\title{
Hesitant Anti-Fuzzy Soft Set in BCK-Algebras
}

\author{
Halimah Alshehri ${ }^{1,2}$ and Noura Alshehri ${ }^{1}$ \\ ${ }^{1}$ Department of Mathematics, Faculty of Science, King Abdulaziz University, Jeddah, Saudi Arabia \\ ${ }^{2}$ Department of Natural and Engineering Science, Faculty of Applied Studies and Community Service, \\ King Saud University, Riyadh, Saudi Arabia \\ Correspondence should be addressed to Halimah Alshehri; haalshehri@ksu.edu.sa
}

Received 6 January 2017; Accepted 30 April 2017; Published 5 June 2017

Academic Editor: Anna M. Gil-Lafuente

Copyright (C) 2017 Halimah Alshehri and Noura Alshehri. This is an open access article distributed under the Creative Commons Attribution License, which permits unrestricted use, distribution, and reproduction in any medium, provided the original work is properly cited.

\begin{abstract}
We introduce the notions of hesitant anti-fuzzy soft set (subalgebras and ideals) and provide relation between them. However, we study new types of hesitant anti-fuzzy soft ideals (implicative, positive implicative, and commutative). Also, we stated and proved some theorems which determine the relationship between these notions.
\end{abstract}

\section{Introduction}

In the real world, there are many complicated problems in economic science, engineering, environment, social science, and management science. They are characterized by uncertainty, imprecision, and vagueness. We cannot successfully utilize the classical methods to deal with these problems because there are various types of uncertainties involved in these problems. Moreover, although there are many theories, such as theory of probability, theory of fuzzy sets, theory of interval mathematics, and theory of rough sets, to be considered as mathematical tools to deal with uncertainties, Molodtsov [1] pointed out that all these theories had their own limitations. Also, in order to overcome these difficulties, Molodtsov [1] firstly proposed a new mathematical tool named soft set theory to deal with uncertainty and imprecision. This theory has been demonstrated to be a useful tool in many applications such as decision-making, measurement theory, and game theory.

The soft set model can be combined with other mathematical models. Maji et al. [2] firstly presented the concept of fuzzy soft set by combining the theories of fuzzy set and soft set together. The hesitant fuzzy set, as one of the extensions of Zadeh's [3] (1965) fuzzy set, allows the membership degree of an element to a set presented by several possible values, and it can express the hesitant information more comprehensively than other extensions of fuzzy set. In [4], Torra introduced the concept of hesitant fuzzy set and Babitha and John (2013) [5] defined another important soft set, hesitant fuzzy soft set. They introduced basic operations such as intersection, union, and compliment, and De Morgan's law was proven. In 2014, Jun et al. [6] applied the notion of hesitant fuzzy soft sets to subalgebras and ideals in BCK/BCI-algebras. In this paper, in Section 3, we introduce the concepts of hesitant anti-fuzzy soft set of subalgebra. In Section 4, we define the hesitant antifuzzy soft ideal in BCK-algebras and give some basic relations. In Section 5, we discuss notion of hesitant anti-fuzzy soft implicative ideals and provide some properties. In Section 6, we investigate concept of hesitant anti-fuzzy soft positive implicative ideals and give some relations. In Section 7, we introduce the notion of hesitant anti-fuzzy soft commutative ideals in BCK-algebras and related properties are investigated. Finally, conclusions are presented in the last section.

\section{Preliminaries}

An algebra $(X ; *, 0)$ of type $(2,0)$ is said to be a BCK-algebra if it satisfies the axioms: for all $x, y, z \in X$,

$$
\begin{aligned}
& (\text { BCK-1) }((x * y) *(x * z)) *(z * y)=0, \\
& (\text { BCK-2) }(x *(x * y)) * y=0, \\
& (\text { BCK-3) } x * x=0 \\
& (\text { BCK-4) } 0 * x=0, \\
& (\text { BCK-5) } x * y=0 \text { and } y * x=0 \text { imply that } x=y .
\end{aligned}
$$


Define a binary relation $\leq$ on $X$ by letting $x \leq y$ if and only if $x * y=0$.

Then $(X ; \leq)$ is a partially ordered set with the least element 0 . In any BCK-algebra $X$, the following hold:

(1) $(x * y) * z=(x * z) * y$.

(2) $x * y \leq x$.

(3) $x * 0=x$.

(4) $(x * z) *(y * z) \leq x * y$.

(5) $x *(x *(x * y))=x * y$.

(6) $x \leq y$ implies that $x * z \leq y * z$ and $z * y \leq z * x$, for all $x, y, z \in X$.

A nonempty subset $I$ of $X$ is called a subalgebra of $X$ if, for any $x, y \in I, x * y \in I$. That is, it is closed under the binary operation $*$ of $X$.

A nonempty subset $I$ of $X$ is called an ideal of $X$ if $\left(I_{1}\right)$ $0 \in I ;\left(I_{2}\right) x * y \in I$ and $y \in I$ imply that $x \in I$.

A nonempty subset $I$ of $X$ is called an implicative ideal if it satisfies $\left(I_{1}\right)$ and $\left(I_{3}\right) x \in I$ whenever $(x *(y * x)) * z \in I$ and $z \in I$.

It is called a commutative ideal if it satisfies $\left(I_{1}\right)$ and $\left(I_{4}\right) x *(y *(y * x)) \in I$ whenever $(x * y) * z \in I$ and $z \in I$; and it is called a positive implicative ideal if it satisfies $\left(I_{1}\right)$ and $\left(I_{5}\right) x * z \in I$ whenever $(x * y) * z \in I$ and $y * z \in I$.

A BCK-algebra $X$ is said to be implicative if it satisfies $\forall x, y \in X: x=x *(y * x)$.

A BCK-algebra $X$ is said to be positive implicative if it satisfies $\forall x, y, z \in X:(x * z) *(y * z)=(x * y) * z$.

A BCK-algebra $X$ is said to be commutative if it satisfies $\forall x, y \in X: x *(x * y)=y *(y * x)$.

Definition 1 (see [3]). Let $S$ be a set. A fuzzy set in $S$ is a function $\mu: S \rightarrow[0,1]$

Definition 2 (see [2]). Let $U$ be an initial universe set and let $E$ be a set of parameters. Let $\mathscr{F}(U)$ denote the set of all fuzzy sets in $U$. Then $(\widetilde{F}, A)$ is called a fuzzy soft set over $U$, where $A \subseteq E$ and $\widetilde{F}$ is a mapping given by $\widetilde{F}: A \rightarrow \mathscr{F}(U)$.

Definition 3 (see $[4,7]$ ). Let $E$ be a reference set. A hesitant fuzzy set on $E$ is defined in terms of a function that when applied to $E$ returns a subset of $[0,1]$ which can be viewed as the following mathematical representation:

$$
H_{E}:=\left\{\left(e, h_{E}(e)\right): e \in E\right\},
$$

where $h_{E}: E \rightarrow p([0,1])$.

Definition 4 (see [7]). Given a nonempty subset $A$ of $X$, a hesitant fuzzy set $H_{X}:=\left\{\left(x ; h_{X}(x): x \in X\right\}\right.$ on $X$ satisfying the condition

$$
h_{X}(x)=\phi ; \quad \forall x \notin A
$$

is called a hesitant fuzzy set related to $A$ (briefly, $A$-hesitant fuzzy set) on $X$ and is represented by $H_{A}:=\left\{\left(x, h_{A}(x)\right): x \in\right.$ $X\} ; h_{A}$ is a mapping from $X$ to $p([0,1])$ with $h_{A}(x)=\phi$, for all $x \notin A$.

\begin{tabular}{ccccc}
\multicolumn{5}{c}{ TABLE 1} \\
\hline$*$ & 0 & $a$ & $b$ & $c$ \\
0 & 0 & 0 & 0 & 0 \\
$a$ & $a$ & 0 & $a$ & 0 \\
$b$ & $b$ & $b$ & 0 & 0 \\
$c$ & $c$ & $b$ & $a$ & 0 \\
\hline
\end{tabular}

Proposition 5 (see [8]). Let $H_{A}:=\left\{\left(x, h_{A}(x)\right) \mid x \in X\right\}$ be an $A$-hesitant anti-fuzzy ideal of $X$. Then the following hold: for all $x, y, z \in A$,

(a) if $x \leq y$, then $h_{A}(x) \subseteq h_{A}(y)$, which means that $h_{A}$ preserves the order,

(b) if $x * y \leq z$, then $h_{A}(x) \subseteq h_{A}(y) \cup h_{A}(z)$.

Proposition 6 (see [9]). In a BCK-algebra $X$, the following hold: for all $x, y, z \in X$,

(i) $((x * z) * z) *(y * z) \leq(x * y) * z$,

(ii) $(x * z) *(x *(x * z))=(x * z) * z$,

(iii) $(x *(y *(y * x))) *(y *(x *(y *(y * x)))) \leq x * y$.

Definition 7 (see $[5,6]$ ). Denote by $H \mathscr{F}(U)$ the set of all hesitant fuzzy sets. A pair $(\widetilde{H}, A)$ is called a hesitant fuzzy soft set over a reference set $U$, where $\widetilde{H}$ is a mapping given by $\widetilde{H}: A \rightarrow H \mathscr{F}(U)$.

\section{Hesitant Anti-Fuzzy Soft Subalgebras}

Definition 8. Given a nonempty subset (subalgebra as much as possible) $A$ of $X$, let $H_{A}:=\left\{\left(x, h_{A}(x)\right) \mid x \in X\right\}$ be an $A$ hesitant fuzzy set on $X$. Then $H_{A}:=\left\{\left(x, h_{A}(x)\right) \mid x \in X\right\}$ is called a hesitant anti-fuzzy subalgebra of $X$ related to $A$ (briefly, $A$-hesitant anti-fuzzy subalgebra of $X$ ) if it satisfies the following condition:

$$
\left(h_{A}(x * y) \subseteq h_{A}(x) \cup h_{A}(y)\right) \quad(\forall x, y \in A) .
$$

An $A$-hesitant anti-fuzzy subalgebra of $X$ with $A=X$ is called a hesitant anti-fuzzy subalgebra of $X$.

Definition 9. For a subset $A$ of $E$, a hesitant fuzzy soft set $(\widetilde{H}, A)$ over $X$ is called a hesitant anti-fuzzy soft subalgebra based on $e \in A$ (briefly, $e$-hesitant anti-fuzzy soft subalgebra) over $X$ if the hesitant fuzzy set,

$$
\widetilde{H}[e]:=\left\{\left(x, h_{\widetilde{H}[e]}(x)\right) \mid x \in X\right\},
$$

on $X$ is a hesitant anti-fuzzy subalgebra of $X$. If $(\widetilde{H}, A)$ is an $e$-hesitant anti-fuzzy soft subalgebra over $X$, for all $e \in A$, we say that $(\widetilde{H}, A)$ is a hesitant anti-fuzzy soft subalgebra.

Example 10. Let $X=\{0, a, b, c\}$ be a BCK-algebra in Table 1 (Cayley).

Consider a set of parameters $E:=\left\{e_{1}, e_{2}, e_{3}, e_{4}\right\}$. Let $(\widetilde{H}, A)$ be a hesitant fuzzy soft set over $X$, where $A=$ $\left\{e_{1}, e_{2}, e_{3}\right\}$, which is given in Table 2 . It is routine to verify that 
TABLE 2: Tabular representation of the hesitant fuzzy soft set $(\widetilde{H}, A)$.

\begin{tabular}{ccccc}
\hline$\widetilde{H}$ & 0 & $a$ & $b$ & $c$ \\
\hline$e_{1}$ & $(0.2,0.3)$ & {$[0.2,0.8]$} & {$[0.2,0.8]$} & {$[0.2,0.8]$} \\
$e_{2}$ & $\{0.3\}$ & $\{0.3\}$ & {$[0.1,0,2) \cup(0,2,0.8]$} & {$[0.1,0.9]$} \\
$e_{3}$ & $\{0.4\}$ & {$[0.3,0.7]$} & {$[0.3,0.5]$} & {$[0.3,0.7]$} \\
\hline
\end{tabular}

$\widetilde{H}\left[e_{1}\right], \widetilde{H}\left[e_{2}\right]$ and $\widetilde{H}\left[e_{3}\right]$ are hesitant anti-fuzzy subalgebra over $X$ based on parameters $e_{1}, e_{2}$, and $e_{3}$. Therefore $(\widetilde{H}, A)$ is a hesitant anti-fuzzy soft subalgebra over $X$.

Proposition 11. If $(\widetilde{H}, A)$ is a hesitant anti-fuzzy soft subalgebra over $X$, then

$$
h_{\widetilde{H}[e]}(0) \subseteq h_{\widetilde{H}[e]}(x), \quad \forall x \in X,
$$

where e is any parameter in $A$.

Proof. For any $x \in X$ and $e \in A$, we have

$$
\begin{aligned}
h_{\widetilde{H}[e]}(0) & =h_{\widetilde{H}[e]}(x * x) \subseteq h_{\widetilde{H}[e]}(x) \cup h_{\widetilde{H}[e]}(x) \\
& =h_{\widetilde{H}[e]}(x) .
\end{aligned}
$$

This completes the proof.

Theorem 12. Let $(\widetilde{H}, A)$ be a hesitant anti-fuzzy soft subalgebra over $X$. If $B \subseteq A$, then $\left(\left.\widetilde{H}\right|_{B}, B\right)$ is a hesitant anti-fuzzy soft subalgebra over $X$.

Proof. Suppose that $(\widetilde{H}, A)$ is a hesitant anti-fuzzy soft subalgebra over $X$. Then $\widetilde{H}_{A}$ is a hesitant anti-fuzzy subalgebra of $X, \forall x \in A$. Since $B \subseteq A, h_{B}(b)$ is a hesitant anti-fuzzy subalgebra of $X, \forall b \in B$. Hence, $\left(\left.\widetilde{H}\right|_{B}, B\right)$ is a hesitant antifuzzy soft subalgebra over $X$.

The following example shows that there exists a hesitant fuzzy soft set $(\widetilde{H}, A)$ over $X$ such that

(i) $(\widetilde{H}, A)$ is not a hesitant anti-fuzzy soft subalgebra over $X$,

(ii) there exists a subset $B$ of $A$ such that $\left(\left.\widetilde{H}\right|_{B}, B\right)$ is a hesitant anti-fuzzy soft subalgebra over $X$.

Example 13. Let $X=\{0, a, b, c\}$ be a BCK-algebra in Table 3 (Cayley).

Consider a set of parameters $A=\left\{e_{1}, e_{2}, e_{3}\right\}$. Let $(\widetilde{H}, A)$ be a hesitant fuzzy soft set over $X$ which is described in Table 4.

Then $\widetilde{H}\left[e_{3}\right]:=\left\{\left(x, h_{\widetilde{H}\left[e_{3}\right]}(x)\right) \mid x \in X\right\}$ on $X$ is not hesitant anti-fuzzy subalgebra of $X$, because

$$
\begin{aligned}
h_{\widetilde{H}\left[e_{3}\right]}(a * a) & =h_{\widetilde{H}\left[e_{3}\right]}(0)=\{0.1\} \nsubseteq(0.1,0.2) \\
& =h_{\widetilde{H}\left[e_{3}\right]}(a) \cup h_{\widetilde{H}\left[e_{3}\right]}(a) .
\end{aligned}
$$

Therefore $(\widetilde{H}, A)$ is not hesitant anti-fuzzy soft subalgebra of $X$. But if we take $B=\left\{e_{1}, e_{2}\right\}$, then $\left(\left.\widetilde{H}\right|_{B}, B\right)$ is a hesitant anti-fuzzy soft subalgebra over $X$.
TABLE 3

\begin{tabular}{lllll}
\hline$*$ & 0 & $a$ & $b$ & $c$ \\
0 & 0 & 0 & 0 & 0 \\
$a$ & $a$ & 0 & $a$ & 0 \\
$b$ & $b$ & $b$ & 0 & 0 \\
$c$ & $c$ & $c$ & $c$ & 0 \\
\hline
\end{tabular}

TABLE 4: Tabular representation of the hesitant fuzzy soft set $(\widetilde{H}, A)$.

\begin{tabular}{ccccc}
\hline$\widetilde{H}$ & 0 & $a$ & $b$ & $c$ \\
\hline$e_{1}$ & $(0.3,0.5)$ & $(0.3,0.8)$ & {$[0.3,0.8]$} & {$[0.3,0.5]$} \\
$e_{2}$ & {$[0.3,0.6)$} & {$[0.3,0.9)$} & {$[0.3,0.8)$} & {$[0.3,0.6]$} \\
$e_{3}$ & $\{0.1\}$ & $(0.1,0.2)$ & {$[0.1,0.3]$} & {$[0.1,0.4]$} \\
\hline
\end{tabular}

Definition 14. Let $(\widetilde{H}, A)$ be a hesitant fuzzy soft set over $X$. For each $\varepsilon \in P([0,1])$, the set $(\widetilde{H}, A)^{\varepsilon}=\left(\widetilde{H}^{\epsilon}, A\right)$ is called a hesitant anti- $\varepsilon$-level soft set of $(\widetilde{H}, A)$, where $\widetilde{H}^{\epsilon}=\{x \in X$ : $\left.h_{\widetilde{H}[e]}(x) \subseteq \varepsilon\right\}$ for all $e \in A$.

Theorem 15. Let $(\widetilde{H}, A)$ be a hesitant fuzzy soft set over $X .(\widetilde{H}, A)$ is a hesitant anti-fuzzy soft subalgebra of $X$ if and only if $(\widetilde{H}, A)^{\varepsilon}$ is a subalgebra over $X$ for each $\varepsilon \in P([0,1])$.

Proof. Suppose that $(\widetilde{H}, A)$ is a hesitant anti-fuzzy soft subalgebra of $X$. For each $\varepsilon \in P([0,1]), e \in A$ and $x_{1}, x_{2} \in \widetilde{H}^{\epsilon}$, $h_{\widetilde{H}[e]}\left(x_{1}\right) \subseteq \varepsilon$ and $h_{\widetilde{H}[e]}\left(x_{2}\right) \subseteq \varepsilon$. Thus

$$
h_{\widetilde{H}[e]}\left(x_{1} * x_{2}\right) \subseteq h_{\widetilde{H}[e]}\left(x_{1}\right) \cup h_{\widetilde{H}[e]}(x) \subseteq \varepsilon .
$$

This implies that $x_{1} * x_{2} \in \widetilde{H}^{\epsilon}$. Hence, $(\widetilde{H}, A)^{\varepsilon}$ is a subalgebra over $X$.

Conversely, assume that $(\widetilde{H}, A)^{\varepsilon}$ is a subalgebra over $X$ for each $\varepsilon \in P([0,1])$. For each $e \in A$ and $x, y \in X$, let $h_{\widetilde{H}[e]}(x)=$ $\varepsilon_{x}$ and let $h_{\widetilde{H}[e]}(y)=\varepsilon_{y}$. Take $\varepsilon=\varepsilon_{x} \cup \varepsilon_{y}$. Then $x, y \in \widetilde{H}^{\epsilon}$ and so $x * y \in \widetilde{H}^{\epsilon}$. Hence,

$$
h_{\widetilde{H}[e]}(x * y) \subseteq \varepsilon=\varepsilon_{x} \cup \varepsilon_{y}=h_{\widetilde{H}[e]}(x) \cup h_{\widetilde{H}[e]}(y) .
$$

Therefore $(\widetilde{H}, A)$ is a hesitant anti-fuzzy subalgebra over $X$. Then, by Definition 8 , we conclude that $(\widetilde{H}, A)$ is a hesitant anti-fuzzy soft subalgebra of $X$. This completes the proof.

\section{Hesitant Anti-Fuzzy Soft Ideals}

Definition 16. Given a nonempty subset (subalgebra as much as possible) $A$ of $X$, let $H_{A}:=\left\{\left(x, h_{A}(x)\right) \mid x \in X\right\}$ be an $A$-hesitant fuzzy set on $X$. Then $H_{A}:=\left\{\left(x, h_{A}(x)\right) \mid x \in X\right\}$ is called a hesitant anti-fuzzy ideal of $X$ related to $A$ (briefly, $A$-hesitant anti-fuzzy ideal of $X$ ) if it satisfies the following conditions:

$$
\begin{aligned}
& (\mathrm{HAFI} 1) h_{A}(0) \subseteq h_{A}(x) . \\
& \left(\text { HAFI2) } h_{A}(x) \subseteq h_{A}(x * y) \cup h_{A}(y) \text { for all } x, y \in A .\right.
\end{aligned}
$$

An $A$-hesitant anti-fuzzy ideal of $X$ with $A=X$ is called a hesitant anti-fuzzy ideal of $X$. 
TABLE 5: Tabular representation of the hesitant fuzzy soft set $(\widetilde{H}, A)$.

\begin{tabular}{lccccc}
\hline$\widetilde{H}$ & Apple & Banana & Carrot & Peach & Radish \\
\hline Cat & $(0.2,0.3)$ & {$[0.2,0.5]$} & {$[0.2,0.5]$} & {$[0.2,0.5]$} & {$[0.2,0.5]$} \\
Cow & {$[0.3,0.4)$} & {$[0.3,0.4)$} & {$[0.3,0.4]$} & {$[0.3,0.4)$} & {$[0.3,0.4)$} \\
Dog & {$[0.1,0.3]$} & {$[0.1,0.7)$} & {$[0.1,0.8)$} & {$[0.1,0.7)$} & {$[0.1,0.7)$} \\
Horse & $\{0.3\}$ & {$[0.3,0.4)$} & {$[0.3,0.4)$} & {$[0.3,0.9)$} & {$[0.3,0.6)$} \\
\hline
\end{tabular}

Definition 17. Let $(\widetilde{H}, A)$ be a hesitant fuzzy soft set over $X$, where $A$ is a subset of $E$. Given $e \in A,(\widetilde{H}, A)$ is called a hesitant anti-fuzzy soft ideal based on $e$ (briefly, $e$-hesitant anti-fuzzy soft ideal) over $X$, if the hesitant fuzzy set,

$$
\widetilde{H}[e]:=\left\{\left(x, h_{\widetilde{H}[e]}(x)\right) \mid x \in X\right\},
$$

on $X$ is a hesitant anti-fuzzy ideal of $X$. If $(\widetilde{H}, A)$ is an $e$ hesitant anti-fuzzy soft ideal over $X$ for all $e \in A$, we say that $(\widetilde{H}, A)$ is a hesitant anti-fuzzy soft ideal over $X$.

Example 18. Let $X:=$ apple, banana, carrot, peach, radish be a reference set, and consider a soft machine $\diamond$ which produces the following products:

apple $\Delta x= \begin{cases}\text { apple } & \text { if } x \in\{\text { carrot, peach, radish }\} \\ \text { carrot } & \text { if } x \in\{\text { apple, banana }\}\end{cases}$

banana $\diamond y$

$= \begin{cases}\text { banana } & \text { if } y \in\{\text { apple, carrot, peach, radish }\} \\ \text { carrot } & \text { if } y=\text { banana }\end{cases}$

peach $\Delta z= \begin{cases}\text { peach } & \text { if } z \in\{\text { carrot, radish }\} \\ \text { carrot } & \text { if } z \in\{\text { apple, banana, peach }\}\end{cases}$

radish $\Delta u= \begin{cases}\text { radish } & \text { if } u \in\{\text { carrot, peach }\} \\ \text { carrot } & \text { if } u \in\{\text { apple, banana, radish }\}\end{cases}$

carrot $\diamond v=$ carrot $\forall v \in X$.

Then $X$ is a BCK-algebra under the soft machine $\diamond$. Consider a set of parameters $A:=\{$ Cat, Cow, Dog, Horse $\}$; let $(\widetilde{H}, A)$ be a hesitant fuzzy soft set over $X$ which is described in Table 5.

It is routine to verify that $(\widetilde{H}, A)$ is a hesitant anti-fuzzy soft ideal over $X$ based on parameters "cat," "cow," and "dog." $\operatorname{But}(\widetilde{H}, A)$ is not a hesitant anti-fuzzy soft ideal of $X$ based on parameter "horse" because

$$
\begin{aligned}
\widetilde{H}[\text { horse }](\text { peach })= & {[0.3,0.9) \nsubseteq[0.3,0.4) } \\
= & \widetilde{H}[\text { horse }](\text { peach } \diamond \text { banana }) \\
& \cup \widetilde{H}[\text { horse }](\text { banana }) .
\end{aligned}
$$

Theorem 19. Let $(\widetilde{H}, A)$ be a hesitant fuzzy soft set over $X .(\widetilde{H}, A)$ is a hesitant anti-fuzzy soft ideal of $X$ if and only if $(\widetilde{H}, A)^{\varepsilon}$ is an ideal over $X$ for each $\varepsilon \in P([0,1])$.
Proof. Suppose that $(\widetilde{H}, A)$ is a hesitant anti-fuzzy soft ideal of $X$. For each $\varepsilon \in P([0,1]), e \in A$ and $x, y \in \widetilde{H}^{\epsilon}$ such that $x * y \in \widetilde{H}^{\epsilon}$ and $y \in \widetilde{H}^{\epsilon}$; then $h_{\widetilde{H}[e]}(x * y) \subseteq \varepsilon$ and $h_{\widetilde{H}[e]}(y) \subseteq \varepsilon$. Thus,

$$
h_{\widetilde{H}[e]}(0) \subseteq h_{\widetilde{H}[e]}(x) \subseteq h_{\widetilde{H}[e]}(x * y) \cup h_{\widetilde{H}[e]}(y) \subseteq \varepsilon .
$$

Hence, $0 \in \widetilde{H}^{\epsilon}$ and $x \in \widetilde{H}^{\epsilon}$, and this implies that $(\widetilde{H}, A)^{\varepsilon}$ is an ideal over $X$.

Conversely, assume that $(\widetilde{H}, A)^{\varepsilon}$ is an ideal over $X$ for each $\varepsilon \in P([0,1])$. For each $e \in A$ and $x, y \in X$, let $h_{\widetilde{H}[e]}(x)=\varepsilon_{x}$, and then $x \in \widetilde{H}^{\epsilon}$. Since $(\widetilde{H}, A)^{\varepsilon}$ is an ideal over $X$, we have $0 \in \widetilde{H}^{\epsilon}$ and so $h_{\widetilde{H}[e]}(0) \subseteq \varepsilon_{x}=h_{\widetilde{H}[e]}(x)$. Let $h_{\widetilde{H}[e]}(x * y)=\varepsilon_{x * y}$ and let $h_{\widetilde{H}[e]}(y)=\varepsilon_{y}$. If we take $\varepsilon=\varepsilon_{x * y} \cup \varepsilon_{y}$, then $x * y \in \widetilde{H}^{\epsilon}$ and $y \in \widetilde{H}^{\epsilon}$ which imply that $x \in \widetilde{H}^{\epsilon}$. Thus,

$$
h_{\widetilde{H}[e]}(x) \subseteq \varepsilon=\varepsilon_{x * y} \cup \varepsilon_{y}=h_{\widetilde{H}[e]}(x * y) \cup h_{\widetilde{H}[e]}(y) .
$$

Therefore $(\widetilde{H}, A)$ is a hesitant anti-fuzzy soft ideal of $X$.

Proposition 20. Every hesitant anti-fuzzy soft ideal $(\widetilde{H}, A)$ over a $B C K / B C I$-algebra $X$ satisfies the following condition for all $e \in A$ and $x, y, z \in X$ :

(a) If $x \leq y$, then $h_{\widetilde{H}[e]}(x) \subseteq h_{\widetilde{H}[e]}(y)$.

(b) $h_{\widetilde{H}[e]}(x * y) \subseteq h_{\widetilde{H}[e]}(x * z) \cup h_{\widetilde{H}[e]}(z * y)$.

(c) If $x * y \leq z$, then $h_{\widetilde{H}[e]}(x) \subseteq h_{\widetilde{H}[e]}(y) \cup h_{\widetilde{H}[e]}(z)$.

(d) If $h_{\widetilde{H}[e]}(x * y)=h_{\widetilde{H}[e]}(0)$, then $h_{\widetilde{H}[e]}(x) \subseteq h_{\widetilde{H}[e]}(y)$.

(e) $h_{\widetilde{H}[e]}(0 *(0 * x)) \subseteq h_{\widetilde{H}[e]}(x)$.

Proof. Let $e \in A$ and $x, y, z \in X$.

(a) If $x \leq y$, then $x * y=0$. Since $(\widetilde{H}, A)$ is a hesitant anti-fuzzy soft ideal of $X$,

$$
\begin{aligned}
h_{\widetilde{H}[e]}(x) & \subseteq h_{\widetilde{H}[e]}(x * y) \cup h_{\widetilde{H}[e]}(y) \\
& =h_{\widetilde{H}[e]}(0) \cup h_{\widetilde{H}[e]}(y)=h_{\widetilde{H}[e]}(y) .
\end{aligned}
$$

(b) Since $(x * y) *(x * z) \leq z * y$, it follows from (a) that $h_{\widetilde{H}[e]}((x * y) *(x * z)) \subseteq h_{\widetilde{H}[e]}(z * y)$. Hence

$$
\begin{aligned}
h_{\widetilde{H}[e]}(x * y) \subseteq & h_{\widetilde{H}[e]}((x * y) *(x * z)) \\
& \cup h_{\widetilde{H}[e]}(x * z) \\
\subseteq & h_{\widetilde{H}[e]}(x * z) \cup h_{\widetilde{H}[e]}(z * y),
\end{aligned}
$$

(c) If $x * y \leq z$, then $(x * y) * z=0$. Since

$$
\begin{aligned}
h_{\widetilde{H}[e]}(z) & =h_{\widetilde{H}[e]}(0) \cup h_{\widetilde{H}[e]}(z) \\
& =h_{\widetilde{H}[e]}((x * y) * z) \cup h_{\widetilde{H}[e]}(z) \\
& \supseteq h_{\widetilde{H}[e]}(x * y),
\end{aligned}
$$

it follows that $h_{\widetilde{H}[e]}(x) \subseteq h_{\widetilde{H}[e]}(x * y) \cup h_{\widetilde{H}[e]}(y) \subseteq h_{\widetilde{H}[e]}(y) \cup$ $h_{\widetilde{H}[e]}(z)$. 
(d) If $h_{\widetilde{H}[e]}(x * y)=h_{\widetilde{H}[e]}(0)$, then we have

$$
\begin{aligned}
h_{\widetilde{H}[e]}(x) & \subseteq h_{\widetilde{H}[e]}(x * y) \cup h_{\widetilde{H}[e]}(y) \\
& =h_{\widetilde{H}[e]}(0) \cup h_{\widetilde{H}[e]}(y)=h_{\widetilde{H}[e]}(y) .
\end{aligned}
$$

(e) For all $x \in X$,

$$
\begin{aligned}
h_{\widetilde{H}[e]}(0 *(0 * x)) \subseteq & h_{\widetilde{H}[e]}((0 *(0 * x)) * x) \\
& \cup h_{\widetilde{H}[e]}(x) \\
= & h_{\widetilde{H}[e]}(0) \cup h_{\widetilde{H}[e]}(x)=h_{\widetilde{H}[e]}(x) .
\end{aligned}
$$

The proof is complete.

Theorem 21. Let $(\widetilde{H}, A)$ be a hesitant fuzzy soft set over $X$ which satisfies condition (5) and (c) from Proposition 20. Then $(\widetilde{H}, A)$ is a hesitant anti-fuzzy soft ideal over $X$.

Proof. Let $e \in A$. Since $x *(x * y) \leq y$, for all $x, y \in X$, it follows from Proposition 20(c) that

$$
h_{\widetilde{H}[e]}(x) \subseteq h_{\widetilde{H}[e]}(x * y) \cup h_{\widetilde{H}[e]}(y) .
$$

Hence, $(\widetilde{H}, A)$ is a hesitant anti-fuzzy soft ideal over $X$.

Theorem 22. Every hesitant anti-fuzzy soft ideal (based on a parameter) over BCK-algebra $X$ is a hesitant anti-fuzzy soft subalgebra (based on the same parameter) over X.

Proof. For any $e \in A$, assume that $(\widetilde{H}, A)$ is a hesitant antifuzzy soft ideal over $X$. Then

$$
\begin{aligned}
h_{\widetilde{H}[e]}(x * y) \subseteq h_{\widetilde{H}[e]}((x * y) * x) \cup h_{\widetilde{H}[e]}(x) \\
=h_{\widetilde{H}[e]}((x * x) * y) \cup h_{\widetilde{H}[e]}(x) \\
=h_{\widetilde{H}[e]}(0 * y) \cup h_{\widetilde{H}[e]}(x) \\
=h_{\widetilde{H}[e]}(0) \cup h_{\widetilde{H}[e]}(x) \\
\subseteq h_{\widetilde{H}[e]}(x) \cup h_{\widetilde{H}[e]}(y),
\end{aligned}
$$

for all $x, y \in X$, and so $(\widetilde{H}, A)$ is a hesitant anti-fuzzy soft subalgebra over $X$.

The following example shows that the converse of Theorem 22 is not true in general.

Example 23. Let $X=\{0, a, b, c\}$ in Example 13 and $B=$ $\left\{e_{1}, e_{2}\right\}$, and then $(\widetilde{H}, B)$ is a hesitant anti-fuzzy soft subalgebra over $X$. But it is not a hesitant anti-fuzzy soft ideal over $X$ based on parameters " $e_{1}$ " and " $e_{2}$ " since

$$
\begin{aligned}
h_{\widetilde{H}\left[e_{1}\right]}(a) & =(0.3,0.8) \nsubseteq[0.3,0.5] \\
& =(0.3,0.5) \cup[0.3,0.5] \\
& =h_{\widetilde{H}\left[e_{1}\right]}(0) \cup h_{\widetilde{H}\left[e_{1}\right]}(c) \\
& =h_{\widetilde{H}\left[e_{1}\right]}(a * c) \cup h_{\widetilde{H}\left[e_{1}\right]}(c) .
\end{aligned}
$$

Also,

$$
\begin{aligned}
h_{\widetilde{H}\left[e_{2}\right]}(a) & =[0.3,0.9) \nsubseteq[0.3,0.6] \\
& =h_{\widetilde{H}\left[e_{2}\right]}(a * c) \cup h_{\widetilde{H}\left[e_{2}\right]}(c) .
\end{aligned}
$$

We provide a condition for a hesitant anti-fuzzy soft subalgebra over $X$ to be a hesitant anti-fuzzy soft ideal over $X$.

Theorem 24. Let $(\widetilde{H}, A)$ be a hesitant anti-fuzzy soft subalgebra over $X$. If the inequality $x * y \leq z$ holds in $X$, then $(\widetilde{H}, A)$ is a hesitant anti-fuzzy soft ideal over $X$.

Proof. Suppose that $(\widetilde{H}, A)$ is a hesitant anti-fuzzy soft subalgebra over $X$. Then, from Proposition 11, we have

$$
h_{\widetilde{H}[e]}(0) \subseteq h_{\widetilde{H}[e]}(x), \quad \forall x \in X, e \in A .
$$

Assume that $x * y \leq z$ holds in $X$. Then, by Proposition 20(c), we get

$$
h_{\widetilde{H}[e]}(x) \subseteq h_{\widetilde{H}[e]}(y) \cup h_{\widetilde{H}[e]}(z) \quad \forall x, y, z \in X
$$

Since $x *(x * y) \leq y$,

$$
h_{\widetilde{H}[e]}(x) \subseteq h_{\widetilde{H}[e]}(x * y) \cup h_{\widetilde{H}[e]}(y) \quad \forall x, y \in X .
$$

Hence, $(\widetilde{H}, A)$ is a hesitant anti-fuzzy soft ideal over $X$.

\section{Hesitant Anti-Fuzzy Soft Implicative Ideals}

Definition 25. A hesitant fuzzy set $H_{X}:=\left\{\left(x, h_{X}(x)\right) \mid\right.$ $x \in X\}$ in a BCK-algebra $X$ is called a hesitant antifuzzy implicative ideal (briefly, HAFII) of $X$ if it satisfies the following conditions:

$$
\begin{aligned}
& \text { (HAFII1) } h_{X}(0) \subseteq h_{X}(x) \text {, for all } x \in X . \\
& \text { (HAFII2) } h_{X}(x) \subseteq h_{X}((x *(y * x)) * z) \cup h_{X}(z) \text {, for } \\
& \text { all } x, y, z \in X .
\end{aligned}
$$

Definition 26. Let $(\widetilde{H}, A)$ be a hesitant fuzzy soft set over $X$, where $A$ is a subset of $E$. Given $e \in A,(\widetilde{H}, A)$ is called a hesitant anti-fuzzy soft implicative ideal based on $e$ (briefly, $e$-hesitant anti-fuzzy soft implicative ideal) over $X$ if the hesitant fuzzy set,

$$
\widetilde{H}[e]:=\left\{\left(x, h_{\widetilde{H}[e]}(x)\right) \mid x \in X\right\},
$$

on $X$ is a hesitant anti-fuzzy implicative ideal of $X$. If $(\widetilde{H}, A)$ is an $e$-hesitant anti-fuzzy soft implicative ideal over $X$, for all $e \in A$, we say that $(\widetilde{H}, A)$ is a hesitant anti-fuzzy soft implicative ideal over $X$.

Proposition 27. Every hesitant anti-fuzzy soft implicative ideal of a BCK-algebra $X$ is order-preserving. 
Proof. Let $(\widetilde{H}, A)$ be a hesitant anti-fuzzy soft implicative ideal over $X$. Let $e \in A$ and $x, y \in X$ be such that $x \leq y$; then

$$
\begin{aligned}
h_{\widetilde{H}[e]}(x) \subseteq h_{\widetilde{H}[e]}((x *(z * x)) * y) \cup h_{\widetilde{H}[e]}(y) \\
=h_{\widetilde{H}[e]}((x * y) *(z * x)) \cup h_{\widetilde{H}[e]}(y) \\
=h_{\widetilde{H}[e]}(0 *(z * x)) \cup h_{\widetilde{H}[e]}(y) \\
=h_{\widetilde{H}[e]}(0) \cup h_{\widetilde{H}[e]}(y)=h_{\widetilde{H}[e]}(y) .
\end{aligned}
$$

Hence, $h_{\widetilde{H}[e]}(x) \subseteq h_{\widetilde{H}[e]}(y)$, and this completes the proof.

Proposition 28. Every hesitant anti-fuzzy soft implicative ideal of a BCK-algebra $X$ is a hesitant anti-fuzzy soft ideal of $X$.

Proof. Let $(\widetilde{H}, A)$ be a hesitant anti-fuzzy soft implicative ideal over $X$. Let $e \in A$ and $x, y, z \in X$; then

$$
h_{\widetilde{H}[e]}(x) \subseteq h_{\widetilde{H}[e]}((x *(y * x)) * z) \cup h_{\widetilde{H}[e]}(z) .
$$

Replace $y=x$, and using $x * x=0$ we get

$$
\begin{aligned}
h_{\widetilde{H}[e]}(x) & \subseteq h_{\widetilde{H}[e]}((x *(x * x)) * z) \cup h_{\widetilde{H}[e]}(z) \\
& =h_{\widetilde{H}[e]}(x * z) \cup h_{\widetilde{H}[e]}(z) .
\end{aligned}
$$

For all $x, z \in X,(\widetilde{H}, A)$ is a hesitant anti-fuzzy soft ideal.

Combining Proposition 28 and Theorem 22 yields the following result.

Corollary 29. Every hesitant anti-fuzzy soft implicative ideal of a BCK-algebra $X$ is a hesitant anti-fuzzy soft subalgebra of $X$.

The following example shows that the converse of Proposition 28 may not be true in general.

Example 30. Let $X=\{0, a, b, c\}$ be a BCK-algebra in Table 6 (Cayley).

Consider a set of parameters $A=\left\{e_{1}, e_{2}\right\}$. Let $(\widetilde{H}, A)$ be a hesitant fuzzy soft set over $X$ which is described in Table 7.

Then $(\widetilde{H}, A)$ is a hesitant anti-fuzzy soft ideal (subalgebra) of $X$. But $\widetilde{H}\left[e_{1}\right]:=\left\{\left(x, h_{\widetilde{H}\left[e_{1}\right]}(x)\right) \mid x \in X\right\}$ and $\widetilde{H}\left[e_{2}\right]:=$ $\left\{\left(x, h_{\widetilde{H}\left[e_{2}\right]}(x)\right) \mid x \in X\right\}$ on $X$ is not hesitant anti-fuzzy soft implicative ideal of $X$, because

$$
\begin{aligned}
h_{\widetilde{H}\left[e_{1}\right]}(a) & =\left[0, \frac{1}{2}\right) \\
& \nsubseteq h_{\widetilde{H}\left[e_{1}\right]}((a *(b * a)) * 0) \cup h_{\widetilde{H}\left[e_{1}\right]}(0) \\
& =h_{\widetilde{H}\left[e_{1}\right]}(0) \cup h_{\widetilde{H}\left[e_{1}\right]}(0)=\left(\frac{1}{4}, \frac{1}{2}\right) .
\end{aligned}
$$

TABLE 6

\begin{tabular}{lllll}
\hline$*$ & 0 & $a$ & $b$ & $c$ \\
0 & 0 & 0 & 0 & 0 \\
$a$ & $a$ & 0 & 0 & $a$ \\
$b$ & $b$ & $a$ & 0 & $b$ \\
$c$ & $c$ & $c$ & $c$ & 0 \\
\hline
\end{tabular}

TABLE 7: Tabular representation of the hesitant fuzzy soft set $(\widetilde{H}, A)$.

\begin{tabular}{lcccc}
\hline$\widetilde{H}$ & 0 & $a$ & $b$ & $c$ \\
\hline$e_{1}$ & $\left(\frac{1}{4}, \frac{1}{2}\right)$ & {$\left[0, \frac{1}{2}\right)$} & {$\left[0, \frac{1}{2}\right)$} & {$\left[0, \frac{3}{4}\right]$} \\
$e_{2}$ & $\left(0, \frac{1}{4}\right)$ & $\left(0, \frac{1}{2}\right)$ & {$\left[0, \frac{1}{2}\right]$} & {$\left[0, \frac{3}{4}\right)$} \\
\hline
\end{tabular}

Also,

$$
\begin{aligned}
h_{\widetilde{H}\left[e_{2}\right]}(a) & =\left(0, \frac{1}{2}\right) \\
& \nsubseteq h_{\widetilde{H}\left[e_{2}\right]}((a *(b * a)) * 0) \cup h_{\widetilde{H}\left[e_{2}\right]}(0) \\
& =h_{\widetilde{H}\left[e_{2}\right]}(0) \cup h_{\widetilde{H}\left[e_{2}\right]}(0)=\left(0, \frac{1}{4}\right) .
\end{aligned}
$$

Now we give a condition for a hesitant anti-fuzzy soft ideal over $X$ to be a hesitant anti-fuzzy soft implicative ideal over $X$.

Theorem 31. If $X$ is an implicative BCK-algebra, then every hesitant anti-fuzzy soft ideal over $X$ is a hesitant anti-fuzzy soft implicative ideal over $X$.

Proof. Let $X$ be an implicative BCK-algebra; it follows that $x=x *(y * x), \forall x, y \in X$. And let $(\widetilde{H}, A)$ be hesitant antifuzzy soft ideal over $X$. Then, for any $e \in A$, we have

$$
\begin{aligned}
h_{\widetilde{H}[e]}(x) & \subseteq h_{\widetilde{H}[e]}(x * z) \cup h_{\widetilde{H}[e]}(z) \\
& =h_{\widetilde{H}[e]}((x *(y * x)) * z) \cup h_{\widetilde{H}[e]}(z)
\end{aligned}
$$

for all $x, y, z \in X$. Hence, it is a hesitant anti-fuzzy implicative ideal of $X$. That is, $(\widetilde{H}, A)$ is hesitant anti-fuzzy soft implicative ideal of $X$.

Theorem 32. Let $(\widetilde{H}, A)$ be a hesitant fuzzy soft set in a BCKalgebra $X$. Then $(\widetilde{H}, A)$ is a hesitant anti-fuzzy soft implicative ideal of $X$ if and only if for each $\varepsilon \in P([0,1])$ the hesitant antifuzzy soft $\varepsilon$-level set $(\widetilde{H}, A)^{\varepsilon}$ of $(\widetilde{H}, A)$ is empty or an implicative ideal of $X$.

Proof. Let $(\widetilde{H}, A)$ be a hesitant anti-fuzzy soft implicative ideal of $X$ and assume that $\widetilde{H}^{\varepsilon} \neq \varnothing$ for every $\varepsilon \in P([0,1])$. Note that $h_{\widetilde{H}[e]}(0) \subseteq h_{\widetilde{H}[e]}(x)$, for all $e \in A$ and $x \in X$. In particular, for each $x \in \widetilde{H}^{\varepsilon}, h_{\widetilde{H}[e]}(0) \subseteq h_{\widetilde{H}[e]}(x) \subseteq \varepsilon$ and so $0 \in \widetilde{H}^{\varepsilon}$. Let $(x *(y * x)) * z \in \widetilde{H}^{\varepsilon}$ and $z \in \widetilde{H}^{\varepsilon}$, and since $(\widetilde{H}, A)$ is a hesitant anti-fuzzy implicative ideal over $X$, it follows that

$$
h_{\widetilde{H}[e]}(x) \subseteq h_{\widetilde{H}[e]}((x *(y * x)) * z) \cup h_{\widetilde{H}[e]}(z) \subseteq \varepsilon .
$$

Hence $x \in \widetilde{H}^{\varepsilon}$, and $\widetilde{H}^{\varepsilon}$ is an implicative ideal of $X$. 
Conversely, let $\widetilde{H}^{\varepsilon}$ be an implicative ideal of $X$; we first show $h_{\widetilde{H}[e]}(0) \subseteq h_{\widetilde{H}[e]}(x)$, for all $e \in A$ and $x \in X$. If not, then there exists $x_{0} \in X$ such that $h_{\widetilde{H}[e]}(0) \supseteq h_{\widetilde{H}[e]}\left(x_{0}\right)$. Let $\varepsilon_{0}=(1 / 2)\left\{h_{\widetilde{H}[e]}(0)+h_{\widetilde{H}[e]}\left(x_{0}\right)\right\}$; then $0 \leq h_{\widetilde{H}[e]}\left(x_{0}\right) \subset \varepsilon_{0} \subset$ $h_{\widetilde{H}[e]}(0) \leq 1$. Thus, $x_{0} \in \widetilde{H}^{\varepsilon_{0}}$ and $\widetilde{H}^{\varepsilon_{0}} \neq \varnothing$. As $\widetilde{H}^{\varepsilon_{0}}$ is an implicative ideal of $X, 0 \in \widetilde{H}^{\varepsilon_{0}}$, which implies that $h_{\widetilde{H}[e]}(0) \subseteq$ $\varepsilon_{0}$. This is a contradiction and so $h_{\widetilde{H}[e]}(0) \subseteq h_{\widetilde{H}[e]}(x)$ for all $e \in A$ and $x \in X$. Now assume that $h_{\widetilde{H}[e]}(x) \subseteq h_{\widetilde{H}[e]}(x *(y *$ $x)) * z) \cup h_{\widetilde{H}[e]}(z)$ does not hold. Then, $h_{\widetilde{H}[e]}\left(x_{0}\right) \supseteq h_{\widetilde{H}[e]}\left(\left(x_{0} *\right.\right.$ $\left.\left.\left(y_{0} * x_{0}\right)\right) * z_{0}\right) \cup h_{\widetilde{H}[e]}\left(z_{0}\right)$ for some $x_{0}, y_{0}, z_{0} \in X$. If we take

$$
\begin{aligned}
\varepsilon_{0} & =\frac{1}{2}\left\{h_{\widetilde{H}[e]}\left(x_{0}\right)\right. \\
& \left.+\left\{h_{\widetilde{H}[e]}\left(\left(x_{0} *\left(y_{0} * x_{0}\right)\right) * z_{0}\right) \cup h_{\widetilde{H}[e]}\left(z_{0}\right)\right\}\right\},
\end{aligned}
$$

then $0 \leq h_{\widetilde{H}[e]}\left(\left(x_{0} *\left(y_{0} * x_{0}\right)\right) * z_{0}\right) \cup h_{\widetilde{H}[e]}\left(z_{0}\right) \subseteq \varepsilon_{0} \subseteq$ $h_{\widetilde{H}[e]}\left(x_{0}\right) \leq 1$. It follows that $h_{\widetilde{H}[e]}\left(\left(x_{0} *\left(y_{0} * x_{0}\right)\right) * z_{0}\right) \subseteq \varepsilon_{0}$ and $h_{\widetilde{H}[e]}\left(z_{0}\right) \subseteq \varepsilon_{0}$, which imply that $\left(x_{0} *\left(y_{0} * x_{0}\right)\right) * z_{0} \in \widetilde{H}^{\varepsilon_{0}}$ and $z_{0} \in \widetilde{H}^{\varepsilon_{0}}$. But $\widetilde{H}^{\varepsilon_{0}}$ is an implicative ideal of $X$, so $x_{0} \in$ $\widetilde{H}^{\varepsilon_{0}}$ or $h_{\widetilde{H}[e]}\left(x_{0}\right) \subseteq \varepsilon_{0}$, and this is a contradiction. Therefore $(\widetilde{H}, A)$ is a hesitant anti-fuzzy soft implicative ideal of $X$.

Theorem 33. Let $(\widetilde{H}, A)$ be a hesitant anti-fuzzy soft ideal of a BCK-algebra $X$. Then $(\widetilde{H}, A)$ is a hesitant anti-fuzzy soft implicative ideal of $X$ if and only if it satisfies the condition

$$
h_{\widetilde{H}[e]}(x) \subseteq h_{\widetilde{H}[e]}(x *(y * x))
$$

for all $x, y \in X$ and $e \in A$.

Proof. Assume that $(\widetilde{H}, A)$ is a hesitant anti-fuzzy soft implicative ideal of $X$. Take $z=0$ in

$$
\begin{aligned}
h_{\widetilde{H}[e]}(x) \subseteq h_{\widetilde{H}[e]}((x *(y * x)) * z) \cup h_{\widetilde{H}[e]}(z) \\
=h_{\widetilde{H}[e]}((x *(y * x)) * 0) \cup h_{\widetilde{H}[e]}(0) \\
=h_{\widetilde{H}[e]}(x *(y * x)) .
\end{aligned}
$$

Conversely, suppose that $(\widetilde{H}, A)$ satisfies the condition. As $(\widetilde{H}, A)$ is a hesitant anti-fuzzy soft ideal of $X$, we have

$$
\begin{aligned}
h_{\widetilde{H}[e]}(x) & \subseteq h_{\widetilde{H}[e]}(x *(y * x)) \\
& \subseteq h_{\widetilde{H}[e]}((x *(y * x)) * z) \cup h_{\widetilde{H}[e]}(z) .
\end{aligned}
$$

Then $(\widetilde{H}, A)$ is a hesitant anti-fuzzy soft implicative ideal of $X$ and the proof is completed.

Now we give characterizations of hesitant anti-fuzzy soft implicative ideals.

Theorem 34. Let $(\widetilde{H}, A)$ be a hesitant anti-fuzzy soft ideal of a BCK-algebra $X$. Then the following are equivalent:

(i) $(\widetilde{H}, A)$ is a hesitant anti-fuzzy soft implicative ideal of $X$.

(ii) $h_{\widetilde{H}[e]}(x) \subseteq h_{\widetilde{H}[e]}(x *(y * x))$ for all $x, y \in X$ and $e \in A$.

(iii) $h_{\widetilde{H}[e]}(x)=h_{\widetilde{H}[e]}(x *(y * x))$ for all $x, y \in X$ and $e \in A$.
Proof. (i) $\Rightarrow$ (ii) Let $(\widetilde{H}, A)$ be a hesitant anti-fuzzy soft implicative ideal of $X$. Then by Theorem 33 we have

$$
\begin{aligned}
h_{\widetilde{H}[e]}(x) & \subseteq h_{\widetilde{H}[e]}((x *(y * x)) * 0) \cup h_{\widetilde{H}[e]}(0) \\
& =h_{\widetilde{H}[e]}(x *(y * x)) \cup h_{\widetilde{H}[e]}(0) \\
& =h_{\widetilde{H}[e]}(x *(y * x)),
\end{aligned}
$$

for all $x, y \in X$. Hence condition (ii) holds.

(ii) $\Rightarrow$ (iii) Observe that, in BCK-algebra $X, x *(y * x) \leq$ $x$. Applying Proposition 20(a), we obtain $h_{\widetilde{H}[e]}(x *(y * x)) \subseteq$ $h_{\widetilde{H}[e]}(x)$. Since (ii) holds, $h_{\widetilde{H}[e]}(x)=h_{\widetilde{H}[e]}(x *(y * x))$ for all $x, y \in X$ and $e \in A$. So, condition (iii) holds.

(iii) $\Rightarrow$ (i) Suppose that condition (iii) holds. Since $(\widetilde{H}, A)$ is a hesitant anti-fuzzy soft ideal of $X$, by Definition 16 , we have

$$
\begin{gathered}
h_{\widetilde{H}[e]}(x *(y * x)) \subseteq h_{\widetilde{H}[e]}((x *(y * x)) * z) \\
\cup h_{\widetilde{H}[e]}(z) .
\end{gathered}
$$

Combining (iii), we obtain

$$
h_{\widetilde{H}[e]}(x) \subseteq h_{\widetilde{H}[e]}((x *(y * x)) * z) \cup h_{\widetilde{H}[e]}(z) .
$$

Thus $(\widetilde{H}, A)$ is a hesitant anti-fuzzy soft implicative ideal of $X$. So, condition (i) holds.

Theorem 35. Let $(\widetilde{H}, A)$ be a hesitant anti-fuzzy soft implicative ideal of a BCK-algebra $X$; then the set

$$
H_{h_{\widetilde{H}[e]}}:=\left\{x \in X \mid h_{\widetilde{H}[e]}(0)=h_{\widetilde{H}[e]}(x)\right\}
$$

is an implicative ideal of $X$.

Proof. Clearly, $0 \in H_{h_{\widetilde{H}[e]}}$. Let $x, y, z \in H_{h_{\widetilde{H}[e]}}$ be such that $(x *(y * x)) * z \in H_{h_{\widetilde{H}[e]}}$ and $z \in H_{h_{\widetilde{H}[e]}}$, for all $e \in A$; then

$$
h_{\widetilde{H}[e]}((x *(y * x)) * z)=h_{\widetilde{H}[e]}(0)=h_{\widetilde{H}[e]}(z) \text {. }
$$

Since $(\widetilde{H}, A)$ is a hesitant anti-fuzzy soft implicative ideal of $X$, it follows that

$$
\begin{aligned}
h_{\widetilde{H}[e]}(x) & \subseteq h_{\widetilde{H}[e]}((x *(y * x)) * z) \cup h_{\widetilde{H}[e]}(z) \\
& =h_{\widetilde{H}[e]}(0) \cup h_{\widetilde{H}[e]}(0)=h_{\widetilde{H}[e]}(0) .
\end{aligned}
$$

Combining Definition 25(HAFII1), we get $h_{\widetilde{H}[e]}(x)=$ $h_{\widetilde{H}[e]}(0)$ and hence $x \in H_{h_{\widetilde{H}[e]}}$. Therefore $H_{h_{\widetilde{H}[e]}}$ is an implicative ideal of $X$.

Theorem 36. A hesitant anti-fuzzy soft subalgebra $h_{\widetilde{H}[e]}$ of $X$ is a hesitant anti-fuzzy soft implicative ideal if and only if it satisfies the condition $(x *(y * x)) * z \leq u$ implying that $h_{\widetilde{H}[e]}(x) \subseteq h_{\widetilde{H}[e]}(z) \cup h_{\widetilde{H}[e]}(u)$ for all $x, y, z, u \in X$ and $\forall e \epsilon$ A.

Proof. Assume that $h_{\widetilde{H}[e]}$ is a hesitant anti-fuzzy soft implicative ideal of $X$ and let $x, y, z, u \in X$ be such that $(x *(y *$ 
$x)) * z \leq u$. Since $h_{\widetilde{H}[e]}$ is also hesitant anti-fuzzy soft ideal of $X$, by Proposition 28, it follows from Proposition 20(c) that

$$
h_{\widetilde{H}[e]}(x *(y * x)) \subseteq h_{\widetilde{H}[e]}(z) \cup h_{\widetilde{H}[e]}(u) .
$$

Making use of Theorem 34, we obtain $h_{\widetilde{H}[e]}(x) \subseteq$ $h_{\widetilde{H}[e]}(z) \cup h_{\widetilde{H}[e]}(u)$.

Conversely, suppose that $h_{\widetilde{H}[e]}$ satisfies the condition in theorem. Obviously, $h_{\widetilde{H}[e]}$ satisfies (5) from Proposition 11. Since $(x *(y * x)) *((x *(y * x)) * z) \leq z$, it follows from Proposition 20(c) and Theorem 34 that

$$
h_{\widetilde{H}[e]}(x) \subseteq h_{\widetilde{H}[e]}((x *(y * x)) * z) \cup h_{\widetilde{H}[e]}(z),
$$

which shows that $h_{\widetilde{H}[e]}$ satisfies conditions of a hesitant antifuzzy implicative ideal of $X$ and so $h_{\widetilde{H}[e]}$ is a hesitant antifuzzy soft implicative ideal of $X$. The proof is complete.

\section{Hesitant Anti-Fuzzy Soft Positive Implicative Ideals}

Definition 37. A hesitant fuzzy set $H_{X}:=\left\{\left(x, h_{X}(x)\right) \mid x \in X\right\}$ in a BCK-algebra $X$ is called a hesitant anti-fuzzy positive implicative ideal (briefly, HAFPII) of $X$ if it satisfies the following conditions:

$$
\begin{aligned}
& \text { (HAFPII1) } h_{X}(0) \subseteq h_{X}(x) \text {, for all } x \in X \\
& \text { (HAFPII2) } h_{X}(x * z) \subseteq h_{X}((x * y) * z) \cup h_{X}(y * z) \text {, } \\
& \text { for all } x, y, z \in X
\end{aligned}
$$

Definition 38. Let $(\widetilde{H}, A)$ be a hesitant fuzzy soft set over $X$, where $A$ is a subset of $E$. Given $e \in A,(\widetilde{H}, A)$ is called a hesitant anti-fuzzy soft positive implicative ideal based on $e$ (briefly, $e$-hesitant anti-fuzzy soft positive implicative ideal) over $X$ if the hesitant fuzzy set,

$$
\widetilde{H}[e]:=\left\{\left(x, h_{\widetilde{H}[e]}(x)\right) \mid x \in X\right\},
$$

on $X$ is a hesitant anti-fuzzy positive implicative ideal of $X$. If $(\widetilde{H}, A)$ is an $e$-hesitant anti-fuzzy soft positive implicative ideal over $X$, for all $e \in A$, we say that $(\widetilde{H}, A)$ is a hesitant anti-fuzzy soft positive implicative ideal over $X$.

Proposition 39. Every hesitant anti-fuzzy soft positive implicative ideal of a BCK-algebra $X$ is order-preserving.

Proof. Let $x, y \in X$ and $e \in A$ be such that $x \leq y$. Since $(\widetilde{H}, A)$ is a hesitant anti-fuzzy soft positive implicative ideal of $X$,

$$
\begin{aligned}
h_{\widetilde{H}[e]}(x * z) \subseteq h_{\widetilde{H}[e]}((x * y) * z) \cup h_{\widetilde{H}[e]}(y * z) \\
=h_{\widetilde{H}[e]}(0 * z) \cup h_{\widetilde{H}[e]}(y * z) \\
=h_{\widetilde{H}[e]}(0) \cup h_{\widetilde{H}[e]}(y * z) \\
=h_{\widetilde{H}[e]}(y * z) .
\end{aligned}
$$

Putting $z=0$,

$$
h_{\widetilde{H}[e]}(x) \subseteq h_{\widetilde{H}[e]}(y) .
$$

The proof is complete.
Proposition 40. Every hesitant anti-fuzzy soft positive implicative ideal of a BCK-algebra $X$ is a hesitant anti-fuzzy soft ideal of $X$.

Proof. Let $(\widetilde{H}, A)$ be a hesitant anti-fuzzy soft positive implicative ideal of a BCK-algebra $X$, so for all $x, y, z \in X$ and $e \in A$ we have

$$
h_{\widetilde{H}[e]}(x * z) \subseteq h_{\widetilde{H}[e]}((x * y) * z) \cup h_{\widetilde{H}[e]}(y * z) .
$$

Putting $z=0$,

$$
h_{\widetilde{H}[e]}(x) \subseteq h_{\widetilde{H}[e]}(x * y) \cup h_{\widetilde{H}[e]}(y) .
$$

Therefore $(\widetilde{H}, A)$ is a hesitant anti-fuzzy soft ideal of $X$.

Combining Theorem 21 and Proposition 39 yields the following result.

Proposition 41. Every hesitant anti-fuzzy soft positive implicative ideal of a BCK-algebra $X$ is a hesitant anti-fuzzy soft subalgebra of $X$.

Remark 42. A hesitant anti-fuzzy soft ideal (subalgebra) of a BCK-algebra $X$ may not be a hesitant anti-fuzzy soft positive implicative ideal of $X$ as shown in the following example.

Example 43. Let $X$ be the BCK-algebra in Example 30. Routine calculations give that $(\widetilde{H}, A)$ is a hesitant anti-fuzzy soft ideal (subalgebra) of $X$, but it is not a hesitant anti-fuzzy soft positive implicative ideal of $X$, because

$$
\begin{aligned}
h_{\widetilde{H}\left[e_{1}\right]}(b * a) & =h_{\widetilde{H}\left[e_{1}\right]}(a)=\left[0, \frac{1}{2}\right) \\
& \nsubseteq h_{\widetilde{H}\left[e_{1}\right]}((b * a) * a) \cup h_{\widetilde{H}\left[e_{1}\right]}(a * a) \\
& =h_{\widetilde{H}\left[e_{1}\right]}(0) \cup h_{\widetilde{H}\left[e_{1}\right]}(0)=\left(\frac{1}{4}, \frac{1}{2}\right) .
\end{aligned}
$$

The converse of Proposition 40 is valid if the following condition holds.

Proposition 44. If $X$ is a positive implicative BCK-algebra, then every hesitant anti-fuzzy soft ideal of $X$ is a hesitant antifuzzy soft positive implicative ideal of $X$.

Proof. Assume that $(\widetilde{H}, A)$ is a hesitant anti-fuzzy soft ideal of a positive implicative BCK-algebra $X$, for all $x, y \in X$ and $e \in A$, and then

$$
h_{\widetilde{H}[e]}(x) \subseteq h_{\widetilde{\mathrm{H}}[e]}(x * y) \cup h_{\widetilde{H}[e]}(y) .
$$

By replacing $x$ by $x * z$ and $y$ by $y * z$, we get

$$
\begin{gathered}
h_{\widetilde{H}[e]}(x * z) \subseteq h_{\widetilde{H}[e]}((x * z) *(y * z)) \\
\cup h_{\widetilde{H}[e]}(y * z) .
\end{gathered}
$$

Since $X$ is a positive implicative BCK-algebra, $(x * z) *$ $(y * z)=(x * y) * z$ for all $x, y, z \in X$. Hence,

$$
h_{\widetilde{H}[e]}(x * z) \subseteq h_{\widetilde{H}[e]}((x * y) * z) \cup h_{\widetilde{H}[e]}(y * z) .
$$


This shows that $(\widetilde{H}, A)$ is a hesitant anti-fuzzy soft positive implicative ideal of $X$.

Theorem 45. Let $(\widetilde{H}, A)$ be a hesitant anti-fuzzy soft ideal over $X$; then $(\widetilde{H}, A)$ is a hesitant anti-fuzzy soft positive implicative ideal of $X$ if and only if

$$
h_{\widetilde{H}[e]}((x * z) *(y * z)) \subseteq h_{\widetilde{H}[e]}((x * y) * z) \text {, }
$$

for alle $\in A$ and $x, y, z \in X$.

Proof. Suppose that $(\widetilde{H}, A)$ is a hesitant anti-fuzzy soft ideal over $X$ and

$$
h_{\widetilde{H}[e]}((x * z) *(y * z)) \subseteq h_{\widetilde{H}[e]}((x * y) * z),
$$

for all $e \in A$ and $x, y, z \in X$.

$$
\begin{aligned}
h_{\widetilde{H}[e]}(x * z) \subseteq & h_{\widetilde{H}[e]}((x * z) *(y * z)) \\
& \cup h_{\widetilde{H}[e]}(y * z) \\
\subseteq & h_{\widetilde{H}[e]}((x * y) * z) \cup h_{\widetilde{H}[e]}(y * z) .
\end{aligned}
$$

Thus $(\widetilde{H}, A)$ is a hesitant anti-fuzzy soft positive implicative ideal of $X$.

Conversely, assume that $(\widetilde{H}, A)$ is a hesitant anti-fuzzy soft positive implicative ideal of $X$ implying that $(\widetilde{H}, A)$ is a hesitant anti-fuzzy soft ideal of $X$ by Proposition 40. Let $a=x *(y * z)$ and let $b=x * y$; since

$$
((x *(y * z)) *(x * y)) \leq y *(y * z),
$$

using Proposition 20(a), we have

$$
\begin{aligned}
& h_{\widetilde{H}[e]}((a * b) * z) \\
& =h_{\widetilde{H}[e]}(((x *(y * z)) *(x * y)) * z) \\
& \subseteq h_{\widetilde{H}[e]}((y *(y * z)) * z)=h_{\widetilde{H}[e]}(0) .
\end{aligned}
$$

And so

$$
\begin{aligned}
& h_{\widetilde{H}[e]}((x * z) *(y * z))=h_{\widetilde{H}[e]}((x *(y * z)) * z) \\
& =h_{\widetilde{H}[e]}(a * z) \subseteq h_{\widetilde{H}[e]}((a * b) * z) \cup h_{\widetilde{H}[e]}(b * z) \\
& \quad \subseteq h_{\widetilde{H}[e]}(0) \cup h_{\widetilde{H}[e]}(b * z)=h_{\widetilde{H}[e]}(b * z) \\
& =h_{\widetilde{H}[e]}((x * y) * z) .
\end{aligned}
$$

Therefore $h_{\widetilde{H}[e]}((x * z) *(y * z)) \subseteq h_{\widetilde{H}[e]}((x * y) * z)$, for all $e \in A$ and $x, y, z \in X$.

Theorem 46. Let $(\widetilde{H}, A)$ be a hesitant anti-fuzzy soft positive implicative ideal of $X$. Then, for all $x, y, z, a, b \in X$ and $e \in A$,

$$
\begin{gathered}
((x * y) * z) * a \leq b \Longrightarrow \\
h_{\widetilde{H}[e]}((x * z) *(y * z)) \subseteq h_{\widetilde{H}[e]}(a) \cup h_{\widetilde{H}[e]}(b) .
\end{gathered}
$$

Proof. Let $x, y, z, a, b \in X$ be such that $((x * y) * z) * a \leq b$. Since $(\widetilde{H}, A)$ is a hesitant anti-fuzzy soft positive implicative ideal of $X$, it follows from Theorem 45 and Proposition 20(c) that

$$
\begin{aligned}
& h_{\widetilde{H}[e]}((x * z) *(y * z)) \subseteq h_{\widetilde{H}[e]}((x * y) * z) \\
& \subseteq h_{\widetilde{H}[e]}(a) \cup h_{\widetilde{H}[e]}(b) .
\end{aligned}
$$

This completes the proof.

Theorem 47. Let $(\widetilde{H}, A)$ be a hesitant anti-fuzzy soft positive implicative ideal of a BCK-algebra $X$, for all $e \in A$. Then the set

$$
H_{h_{\widetilde{H}[e]}}:=\left\{x \in X \mid h_{\widetilde{H}[e]}(0)=h_{\widetilde{H}[e]}(x)\right\}
$$

is a positive implicative ideal of $X$.

Proof. Let $e \in A$. Clearly, $0 \in H_{h_{\widetilde{H}[e]}}$. Let $x, y, z \in H_{h_{\widetilde{H}[e]}}$ be such that $(x * y) * z \in H_{h_{\widetilde{H}[e]}}$ and $y * z \in H_{h_{\widetilde{H}[e]}}$, and then

$$
h_{\widetilde{H}[e]}((x * y) * z)=h_{\widetilde{H}[e]}(0)=h_{\widetilde{H}[e]}(y * z) \text {. }
$$

It follows that

$$
\begin{aligned}
h_{\widetilde{H}[e]}(x * z) \subseteq h_{\widetilde{H}[e]}((x * y) * z) \cup h_{\widetilde{H}[e]}(y * z) \\
=h_{\widetilde{H}[e]}(0) \cup h_{\widetilde{H}[e]}(0)=h_{\widetilde{H}[e]}(0),
\end{aligned}
$$

since $(\widetilde{H}, A)$ is a hesitant anti-fuzzy positive implicative ideal of $X$, and then $h_{\widetilde{H}[e]}(x * z)=h_{\widetilde{H}[e]}(0)$ and hence $x * z \epsilon$ $H_{h_{\widetilde{H}[e]}}$. Therefore $H_{h_{\widetilde{H}[e]}}$ is a positive implicative ideal of $X$.

Theorem 48. Let $(\widetilde{H}, A)$ be a hesitant anti-fuzzy soft ideal over $X$; then $(\widetilde{H}, A)$ is a hesitant anti-fuzzy soft positive implicative ideal of $X$ if and only if it satisfies the inequalities

$$
\begin{aligned}
& h_{\widetilde{H}[e]}(x * y) \subseteq h_{\widetilde{H}[e]}((x * y) * y) \\
& \forall x, y \in X, e \in A,
\end{aligned}
$$

for all $e \in A$ and $x, y, z \in X$.

Proof. Suppose that the hesitant anti-fuzzy soft ideal $(\widetilde{H}, A)$ of a BCK-algebra $X$ is a hesitant anti-fuzzy soft filter positive implicative ideal of $X$. So

$$
\begin{array}{r}
h_{\widetilde{H}[e]}(x * z) \subseteq h_{\widetilde{H}[e]}((x * y) * z) \cup h_{\widetilde{H}[e]}(y * z) \\
\forall x, y, z \in X, e \in A .
\end{array}
$$

Substituting $z=y$, we have

$$
\begin{aligned}
h_{\widetilde{H}[e]}(x * y) \subseteq h_{\widetilde{H}[e]}((x * y) * y) \cup h_{\widetilde{H}[e]}(y * y) \\
=h_{\widetilde{H}[e]}((x * y) * y) \cup h_{\widetilde{H}[e]}(0) \\
=h_{\widetilde{H}[e]}((x * y) * y),
\end{aligned}
$$

for all $x, y \in X$ and $e \in A$. 
Conversely, suppose that $(\widetilde{H}, A)$ is a hesitant anti-fuzzy soft ideal over $X$ and satisfies inequality (67).

$$
\begin{aligned}
& h_{\widetilde{H}[e]}(x * y) \subseteq h_{\widetilde{H}[e]}((x * y) * y) \\
& \forall x, y \in X, e \in A,
\end{aligned}
$$

since $h_{\widetilde{H}[e]}(0) \subseteq h_{\widetilde{H}[e]}(x)$. Now we can prove that $h_{\widetilde{H}[e]}(x *$ $z) \subseteq h_{\widetilde{H}[e]}((x * y) * z) \cup h_{\widetilde{H}[e]}(y * z)$ for all $x, y, z \in X$ and $e \in A$. In contrast, there exist $\dot{x}, \grave{y} \in X$ such that

$$
\begin{aligned}
h_{\widetilde{H}[e]}(\grave{x} * \grave{y}) & \supseteq h_{\widetilde{H}[e]}((\grave{x} * \grave{y}) * \grave{y}) \cup h_{\widetilde{H}[e]}(\grave{y} * \grave{y}) \\
& =h_{\widetilde{H}[e]}((\grave{x} * \grave{y}) * \grave{y}) \cup h_{\widetilde{H}[e]}(0) \\
& =h_{\widetilde{H}[e]}((\grave{x} * \grave{y}) * \grave{y}),
\end{aligned}
$$

which is a contradiction. Therefore $h_{\widetilde{H}[e]}(x * z) \subseteq h_{\widetilde{H}[e]}((x *$ $y) * z) \cup h_{\widetilde{H}[e]}(y * z)$ for all $x, y, z \in X$ and $e \in A$. Thus $(\widetilde{H}, A)$ is a hesitant anti-fuzzy soft positive implicative ideal of $X$.

Now we give the condition that makes equality in Theorem 48 hold.

Theorem 49. If $X$ is positive implicative, then a hesitant antifuzzy soft ideal of $X$ is a hesitant anti-fuzzy soft positive implicative ideal of $X$ if and only if it satisfies

$$
\begin{aligned}
& h_{\widetilde{H}[e]}(x * y)=h_{\widetilde{H}[e]}((x * y) * y) \\
& \forall x, y \in X, e \in A .
\end{aligned}
$$

Proof. Suppose that the hesitant anti-fuzzy soft ideal $(\widetilde{H}, A)$ of a BCK-algebra $X$ is a hesitant anti-fuzzy soft positive implicative ideal of $X$. So by Theorem 48 we have

$$
h_{\widetilde{H}[e]}(x * y) \subseteq h_{\widetilde{H}[e]}((x * y) * y)
$$

$$
\forall x, y \in X, e \in A .
$$

On the other hand, since $(x * y) * y \leq x * y$, it follows from Proposition 20 (a) that $h_{\widetilde{H}[e]}((x * y) * y) \subseteq h_{\widetilde{H}[e]}(x * y)$. Thus we have

$$
\begin{aligned}
& h_{\widetilde{H}[e]}(x * y)=h_{\widetilde{H}[e]}((x * y) * y) \\
& \forall x, y \in X, e \in A .
\end{aligned}
$$

Conversely, assume that $h_{\widetilde{H}[e]}(x * y)=h_{\widetilde{H}[e]}((x * y) * y)$ for all $x, y \in X$ and $e \in A$. We want to prove that a hesitant anti-fuzzy soft ideal $(\widetilde{H}, A)$ of a BCK-algebra $X$ is a hesitant anti-fuzzy soft positive implicative ideal of $X$. It is clear that $h_{\widetilde{H}[e]}(0) \subseteq h_{\widetilde{H}[e]}(x)$ for all $x \in X$ and $e \in A$. Since $(\widetilde{H}, A)$ is a hesitant anti-fuzzy soft ideal and $X$ is positive implicative,

$$
\begin{aligned}
h_{\widetilde{H}[e]}(x * z)= & h_{\widetilde{H}[e]}((x * z) * z) \\
\subseteq & h_{\widetilde{H}[e]}((x * z) *(y * z)) \\
& \cup h_{\widetilde{H}[e]}(y * z) \\
= & h_{\widetilde{H}[e]}((x * y) * z) \cup h_{\widetilde{H}[e]}(y * z),
\end{aligned}
$$

for all $x, y, z \in X$ and $e \in A$. Therefore a hesitant anti-fuzzy soft ideal $(\widetilde{H}, A)$ of $X$ is a hesitant anti-fuzzy soft positive implicative ideal of $X$.

Theorem 50. Let $(\widetilde{H}, A)$ be a hesitant anti-fuzzy soft positive implicative ideal of $X$ if and only if for each $\varepsilon \in P([0,1])$ the hesitant anti-fuzzy soft $\varepsilon$-level set $(\widetilde{H}, A)^{\varepsilon}$ of $(\widetilde{H}, A)$ is a positive implicative ideal of $X$.

Proof. Suppose that $(\widetilde{H}, A)$ is a hesitant anti-fuzzy soft positive implicative ideal of $X$ and for every $\varepsilon \in P([0,1])$ define the sets

$$
(\widetilde{H}, A)^{\varepsilon}:=\left\{x \in X \mid h_{\widetilde{H}[e]}(x) \subseteq \varepsilon\right\} \quad \forall e \in A .
$$

Since $(\widetilde{H}, A)^{\varepsilon} \neq \varnothing$, let $x \in(\widetilde{H}, A)^{\varepsilon} \Rightarrow h_{\widetilde{H}[e]}(x) \subseteq \varepsilon$. By Definition 37, we have $h_{\widetilde{H}[e]}(0) \subseteq h_{\widetilde{H}[e]}(x)$, for all $e \in A$ and $x \in X$, implying that $0 \in(\widetilde{H}, A)^{\varepsilon}$. Let $x, y, z \in X$ be such that $(x * y) * z \in(\widetilde{H}, A)^{\varepsilon}$ and $y * z \in(\widetilde{H}, A)^{\varepsilon}$, implying that $h_{\widetilde{H}[e]}((x * y) * z) \subseteq \varepsilon$ and $h_{\widetilde{H}[e]}(y * z) \subseteq \varepsilon$. Since $(\widetilde{H}, A)$ is a hesitant anti-fuzzy soft positive implicative ideal over $X$, it follows that

$$
\begin{aligned}
h_{\widetilde{H}[e]}(x * z) \subseteq h_{\widetilde{H}[e]}((x * y) * z) \cup h_{\widetilde{H}[e]}(y * z) \\
\subseteq \mathcal{\varepsilon} .
\end{aligned}
$$

Hence $x * z \in(\widetilde{H}, A)^{\varepsilon}$, and $(\widetilde{H}, A)^{\varepsilon}$ is a positive implicative ideal of $X$.

Conversely, suppose that $(\widetilde{H}, A)^{\varepsilon}$ is a positive implicative ideal of $X$ for all $\varepsilon \in P([0,1])$. Put $h_{\widetilde{H}[e]}(x)=\varepsilon$ for any $x \in X$. Hence $0 \in(\widetilde{H}, A)^{\varepsilon} \Rightarrow h_{\widetilde{H}[e]}(0) \subseteq \varepsilon=h_{\widetilde{H}[e]}(x)$, for all $e \in A$ and $x \in X$. Now we prove that $h_{\widetilde{H}[e]}(x * z) \subseteq h_{\widetilde{H}[e]}((x * y) *$ $z) \cup h_{\widetilde{H}[e]}(y * z)$. In contrast, there exists $x_{0}, y_{0}, z_{0} \in X$ such that $h_{\widetilde{H}[e]}\left(x_{0} * z_{0}\right) \supseteq h_{\widetilde{H}[e]}\left(\left(x_{0} * y_{0}\right) * z_{0}\right) \cup h_{\widetilde{H}[e]}\left(y_{0} * z_{0}\right)$. Taking

$$
\begin{aligned}
\varepsilon_{0} & =\frac{1}{2}\left\{h_{\widetilde{H}[e]}\left(x_{0} * z_{0}\right)\right. \\
& \left.+\left\{h_{\widetilde{H}[e]}\left(\left(x_{0} * y_{0}\right) * z_{0}\right) \cup h_{\widetilde{H}[e]}\left(y_{0} * z_{0}\right)\right\}\right\},
\end{aligned}
$$

it follows that $h_{\widetilde{H}[e]}\left(\left(x_{0} * y_{0}\right) * z_{0}\right) \subseteq \varepsilon_{0}$ and $h_{\widetilde{H}[e]}\left(y_{0} * z_{0}\right) \subseteq \varepsilon_{0}$, which imply that $\left(x_{0} * y_{0}\right) * z_{0} \in(\widetilde{H}, A)^{\varepsilon_{0}}$ and $y_{0} * z_{0} \epsilon$ $(\widetilde{H}, A)^{\varepsilon_{0}}$. But $(\widetilde{H}, A)^{\varepsilon_{0}}$ is a positive implicative ideal of $X$, and thus $x_{0} * z_{0} \in(\widetilde{H}, A)^{\varepsilon_{0}}$ or $h_{\widetilde{H}[e]}\left(x_{0} * z_{0}\right) \subseteq \varepsilon_{0}$, and this is a contradiction. Therefore

$$
\begin{array}{r}
h_{\widetilde{H}[e]}(x * z) \subseteq h_{\widetilde{H}[e]}((x * y) * z) \cup h_{\widetilde{H}[e]}(y * z) \\
\forall x, y, z \in X, e \in A .
\end{array}
$$

Hence $(\widetilde{H}, A)$ is a hesitant anti-fuzzy soft positive implicative ideal of $X$.

\section{Hesitant Anti-Fuzzy Soft Commutative Ideals}

Definition 51. A hesitant fuzzy set $H_{X}:=\left\{\left(x, h_{X}(x)\right) \mid\right.$ $x \in X\}$ in a BCK-algebra $X$ is called a hesitant anti-fuzzy 
commutative ideal (briefly, HAFCI) of $X$ if it satisfies the following conditions:

(HAFCI1) $h_{X}(0) \subseteq h_{X}(x)$, for all $x \in X$.

$(\mathrm{HAFCI} 2) h_{X}(x *(y *(y * x))) \subseteq h_{X}((x * y) * z) \cup$ $h_{X}(z)$, for all $x, y, z \in X$.

Definition 52. Let $(\widetilde{H}, A)$ be a hesitant fuzzy soft set over $X$, where $A$ is a subset of $E$. Given $e \in A,(\widetilde{H}, A)$ is called a hesitant anti-fuzzy soft commutative ideal based on $e$ (briefly, $e$-hesitant anti-fuzzy soft commutative ideal) over $X$ if the hesitant fuzzy set,

$$
\widetilde{H}[e]:=\left\{\left(x, h_{\widetilde{H}[e]}(x)\right) \mid x \in X\right\},
$$

on $X$ is a hesitant anti-fuzzy commutative ideal of $X$. If $(\widetilde{H}, A)$ is an $e$-hesitant anti-fuzzy soft commutative ideal over $X$, for all $e \in A$, we say that $(\widetilde{H}, A)$ is a hesitant anti-fuzzy soft commutative ideal over $X$.

Proposition 53. Every hesitant anti-fuzzy soft commutative ideal of a BCK-algebra $X$ is order-preserving.

Proof. Let $x, y \in X$ and $e \in A$ be such that $x \leq y$. Since $(\widetilde{H}, A)$ is a hesitant anti-fuzzy soft commutative ideal of $X$,

$$
\begin{aligned}
& h_{\widetilde{H}[e]}(x *(y *(y * x))) \\
& \quad \subseteq h_{\widetilde{H}[e]}((x * y) * z) \cup h_{\widetilde{H}[e]}(z) \\
& \quad=h_{\widetilde{H}[e]}(0 * z) \cup h_{\widetilde{H}[e]}(z)=h_{\widetilde{H}[e]}(0) \cup h_{\widetilde{H}[e]}(z) \\
& \quad=h_{\widetilde{H}[e]}(z) .
\end{aligned}
$$

Putting $y=0$ and replacing $z=y$, we obtain

$$
h_{\widetilde{H}[e]}(x) \subseteq h_{\widetilde{H}[e]}(y) .
$$

The proof is complete.

Theorem 54. Any hesitant anti-fuzzy soft commutative ideal of BCK-algebra $X$ is hesitant anti-fuzzy soft ideal of $X$.

Proof. Let $x, y, z \in X$ and let $e \in A$. And let $(\widetilde{H}, A)$ be a hesitant anti-fuzzy soft commutative ideal of a BCK-algebra $X$, so we have

$$
\begin{aligned}
h_{\widetilde{H}[e]}(x) & =h_{\widetilde{H}[e]}(x *(0 *(0 * x))) \\
& \subseteq h_{\widetilde{H}[e]}((x * 0) * z) \cup h_{\widetilde{H}[e]}(z) \\
& =h_{\widetilde{H}[e]}(x * z) \cup h_{X}(z),
\end{aligned}
$$

for all $x, z \in X$ and $e \in A$. Hence $(\widetilde{H}, A)$ is a hesitant antifuzzy soft ideal of $X$. result.

Combining Theorems 22 and 54 yields the following

Corollary 55. Every hesitant anti-fuzzy soft commutative ideal of a BCK-algebra $X$ is a hesitant anti-fuzzy soft subalgebra of $X$.
TABLE 8

\begin{tabular}{llll}
\hline$*$ & 0 & $a$ & $b$ \\
0 & 0 & 0 & 0 \\
$a$ & $a$ & 0 & 0 \\
$b$ & $b$ & $b$ & 0 \\
\hline
\end{tabular}

TABle 9: Tabular representation of the hesitant fuzzy soft set $(\widetilde{H}, A)$.

\begin{tabular}{lccc}
\hline$\widetilde{H}$ & 0 & $a$ & $b$ \\
\hline$e_{1}$ & $(0.2,0.4)$ & {$[0.2,0.7)$} & {$[0,1)$} \\
$e_{2}$ & $(0,0.2)$ & $(0,0.5)$ & {$[0,0.7)$} \\
\hline
\end{tabular}

Remark 56. A hesitant anti-fuzzy soft ideal (subalgebra) of a BCK-algebra $X$ may not be a hesitant anti-fuzzy soft commutative ideal of $X$ as shown in the following example.

Example 57. Let $X=\{0, a, b\}$ be the BCK-algebra with in Table 8 (Cayley).

Consider a set of parameters $A=\left\{e_{1}, e_{2}\right\}$. Let $(\widetilde{H}, A)$ be a hesitant fuzzy soft set over $X$ which is described in Table 9.

Then $(\widetilde{H}, A)$ is a hesitant anti-fuzzy soft ideal (subalgebra) of $X$. Routine calculations give that $(\widetilde{H}, A)$ is a hesitant anti-fuzzy soft ideal (subalgebra) of $X$, but $\widetilde{H}\left[e_{1}\right]:=$ $\left\{\left(x, h_{\widetilde{H}\left[e_{1}\right]}(x)\right) \mid x \in X\right\}$ and $\widetilde{H}\left[e_{2}\right]:=\left\{\left(x, h_{\widetilde{H}\left[e_{2}\right]}(x)\right) \mid x \in X\right\}$ on $X$ is not hesitant anti-fuzzy soft commutative ideal of $X$, because

$$
\begin{aligned}
& h_{\widetilde{H}\left[e_{1}\right]}(a *(b *(b * a)))=[0.2,0.7) \nsubseteq(0.2,0.4) \\
& =h_{\widetilde{H}\left[e_{1}\right]}((a * b) * 0) \cup h_{\widetilde{H}\left[e_{1}\right]}(0) .
\end{aligned}
$$

Also,

$$
\begin{gathered}
h_{\widetilde{H}\left[e_{2}\right]}(a *(b *(b * a)))=(0,0.5) \nsubseteq(0,0.2) \\
=h_{\widetilde{H}\left[e_{2}\right]}((a * b) * 0) \cup h_{\widetilde{H}\left[e_{2}\right]}(0)
\end{gathered}
$$

The condition in the following theorem makes converse Theorem 54 valid.

Theorem 58. In a commutative BCK-algebra X. Every hesitant anti-fuzzy soft ideal of $X$ is a hesitant anti-fuzzy soft commutative ideal of $X$.

Proof. Suppose that $(\widetilde{H}, A)$ is a hesitant anti-fuzzy soft ideal of a BCK-algebra $X$. It is sufficient to show that $(\widetilde{H}, A)$ satisfies condition (HAFCI2). Let $x, y, z \in X$. Then

$$
\begin{aligned}
& ((x *(y *(y * x))) *((x * y) * z)) * z \\
& \quad=((x *(y *(y * x))) * z) *((x * y) * z) \\
& \quad \leq(x *(y *(y * x))) *(x * y) \\
& \quad=(x *(x * y)) *(y *(y * x))=0 ;
\end{aligned}
$$

that is,

$$
(x *(y *(y * x))) *((x * y) * z) \leq z .
$$


It follows from Proposition 20(c) that

$$
\begin{aligned}
& h_{\widetilde{H}[e]}(x *(y *(y * x))) \\
& \quad \subseteq h_{\widetilde{H}[e]}((x * y) * z) \cup h_{\widetilde{H}[e]}(z),
\end{aligned}
$$

for all $x, y, z \in X$ and $e \in A$. Thus $(\widetilde{H}, A)$ is a hesitant antifuzzy soft commutative ideal of a BCK-algebra $X$, and the proof is complete.

Theorem 59. Let $(\widetilde{H}, A)$ be a hesitant anti-fuzzy soft ideal of a BCK-algebra $X$. Then $(\widetilde{H}, A)$ is a hesitant anti-fuzzy soft commutative ideal of $X$ if and only if it satisfies the condition

$$
\begin{aligned}
& h_{\widetilde{H}[e]}(x *(y *(y * x))) \subseteq h_{\widetilde{H}[e]}(x * y) \\
& \forall x, y \in X, e \in A .
\end{aligned}
$$

Proof. Let $x, y, z \in X$ and let $e \in A$. And suppose that $(\widetilde{H}, A)$ is a hesitant anti-fuzzy soft commutative ideal of $X$. Taking $z=0$, we have

$$
\begin{aligned}
& h_{\widetilde{H}[e]}(x *(y *(y * x))) \\
& \quad \subseteq h_{\widetilde{H}[e]}((x * y) * 0) \cup h_{\widetilde{H}[e]}(0)=h_{\widetilde{H}[e]}(x * y) .
\end{aligned}
$$

Conversely, assume that $(\widetilde{H}, A)$ satisfies condition (89) as $(\widetilde{H}, A)$ is a hesitant anti-fuzzy soft ideal of a BCK-algebra $X$. Hence

$$
\begin{aligned}
& h_{\widetilde{H}[e]}(x * y) \subseteq h_{\widetilde{H}[e]}((x * y) * z) \cup h_{\widetilde{H}[e]}(z) \\
& \forall x, y, z \in X, e \in A .
\end{aligned}
$$

Combining (91) and (89), we obtain

$$
\begin{aligned}
& h_{\widetilde{H}[e]}(x *(y *(y * x))) \\
& \quad \subseteq h_{\widetilde{H}[e]}((x * y) * z) \cup h_{\widetilde{H}[e]}(z),
\end{aligned}
$$

for all $x, y, z \in X$ and $e \in A$. Hence $(\widetilde{H}, A)$ is hesitant antifuzzy soft commutative ideal of $X$, which completes the proof.

Observing $x * y \leq x *(y *(y * x))$ and using Proposition 20(a), we have $h_{\widetilde{H}[e]}(x * y) \subseteq h_{\widetilde{H}[e]}(x *(y *(y *$ $x))$ ) for all $x, y \in X$ and $e \in A$. Hence, Theorem 59 can be improved as follows.

Theorem 60. A hesitant anti-fuzzy soft ideal $(\widetilde{H}, A)$ of a BCKalgebra $X$ is a hesitant anti-fuzzy soft commutative ideal of $X$ if and only if it satisfies the identity

$$
\begin{array}{r}
h_{\widetilde{H}[e]}(x * y)=h_{\widetilde{H}[\mathrm{e}]}(x *(y *(y * x))) \\
\quad \forall x, y \in X, e \in A .
\end{array}
$$

Now we describe a relation between hesitant anti-fuzzy soft implicative ideals, hesitant anti-fuzzy soft commutative ideals, and hesitant anti-fuzzy soft positive implicative ideals.
Theorem 61. A hesitant anti-fuzzy soft ideal $(\widetilde{H}, A)$ of a BCKalgebra $X$ is a hesitant anti-fuzzy soft implicative ideal if and only if $(\widetilde{H}, A)$ is both hesitant anti-fuzzy soft commutative ideal and hesitant anti-fuzzy soft positive implicative ideal.

Proof. Assume that $(\widetilde{H}, A)$ is a hesitant anti-fuzzy soft implicative ideal. By Propositions 6(i) and 20(c), for all $x, y, z \in X$ and $e \in A$, we have

$$
\begin{aligned}
& h_{\widetilde{H}[e]}((x * y) * z) \cup h_{\widetilde{H}[e]}(y * z) \\
& \supseteq h_{\widetilde{H}[e]}((x * z) * z) \\
&=h_{\widetilde{H}[e]}((x * z)) \\
& \quad *(x *(x * z)), \quad[\text { by Proposition } 6(\mathrm{ii})] \\
&=h_{\widetilde{H}[e]}(x * z), \quad[\text { by Theorem } 34 \text { (iii)]. }
\end{aligned}
$$

Hence $(\widetilde{H}, A)$ is a hesitant anti-fuzzy soft positive implicative ideal of $X$.

Also, By Propositions 20 and 6(iii) and Theorem 34(iii), we have

$$
\begin{aligned}
h_{\widetilde{H}[e]} & (x *(y *(y * x))) \\
= & h_{\widetilde{H}[e]}((x *(y *(y * x)))) \\
& *(y *(x *(y *(y * x)))) \subseteq h_{\widetilde{H}[e]}(x * y) .
\end{aligned}
$$

It follows from Theorem 59 that $(\widetilde{H}, A)$ is hesitant antifuzzy soft commutative ideal of $X$.

Conversely, suppose that $(\widetilde{H}, A)$ is both hesitant antifuzzy soft positive implicative ideal of $X$ and hesitant antifuzzy soft commutative ideal of $X$. Since $(y *(y * x)) *(y * x) \leq$ $x *(y * x)$, it follows from Proposition 20(a) that

$$
h_{\widetilde{H}[e]}((y *(y * x)) *(y * x)) \subseteq h_{\widetilde{H}[e]}(x *(y * x))
$$

Using Theorem 49, we have $h_{\widetilde{H}[e]}((y *(y * x)) *(y * x))=$ $h_{\widetilde{H}[e]}(y *(y * x))$ and so

$$
h_{\widetilde{H}[e]}(y *(y * x)) \subseteq h_{\widetilde{H}[e]}(x *(y * x)) .
$$

On the other hand, $x * y \leq x *(y * x)$ implies that $h_{\widetilde{H}[e]}(x *$ $y) \subseteq h_{\widetilde{H}[e]}(x *(y * x))$. Since $(\widetilde{H}, A)$ is a hesitant anti-fuzzy soft commutative ideal of $X$, By Theorem 60 , we have

$$
h_{\widetilde{H}[e]}(x * y)=h_{\widetilde{H}[e]}(x *(y *(y * x))) .
$$

Hence,

$$
h_{X}(x *(y *(y * x))) \subseteq h_{X}(x *(y * x)) .
$$

Combining (97), we obtain

$$
\begin{aligned}
h_{\widetilde{H}[e]}(x *(y * x)) \supseteq h_{\widetilde{H}[e]}(x *(y *(y * x))) \\
\cup h_{\widetilde{H}[e]}(y *(y * x)) \\
\supseteq h_{\widetilde{H}[e]}(x) .
\end{aligned}
$$

So, $(\widetilde{H}, A)$ is a hesitant anti-fuzzy soft implicative ideal of $X$ by Theorem 34 . The proof is complete. 
Theorem 62. Let $(\widetilde{H}, A)$ be hesitant anti-fuzzy soft commutative ideal of $X$ if and only if for each $\varepsilon \in P([0,1])$ the hesitant anti-fuzzy soft $\varepsilon$-level set $(\widetilde{H}, A)^{\varepsilon}$ of $(\widetilde{H}, A)$ is a commutative ideal of $X$.

Proof. Suppose that $(\widetilde{H}, A)$ is a hesitant anti-fuzzy soft commutative ideal of $X$ and for every $\varepsilon \in P([0,1])$ define the sets

$$
(\widetilde{H}, A)^{\varepsilon}:=\left\{x \in X \mid h_{\widetilde{H}[e]}(x) \subseteq \varepsilon\right\} \quad \forall e \in A .
$$

Since $(\widetilde{H}, A)^{\varepsilon} \neq \varnothing$, let $x \in(\widetilde{H}, A)^{\varepsilon} \Rightarrow h_{\widetilde{H}[e]}(x) \subseteq \varepsilon$. By definition, we have $h_{\widetilde{H}[e]}(0) \subseteq h_{\widetilde{H}[e]}(x)$, for all $e \in A$ and $x \in X$, implying that $0 \in(\widetilde{H}, A)^{\varepsilon}$. Let $x, y, z \in X$ be such that $(x * y) * z \in(\widetilde{H}, A)^{\varepsilon}$ and $z \in(\widetilde{H}, A)^{\varepsilon}$, implying that $h_{\widetilde{H}[e]}((x * y) * z) \subseteq \varepsilon$ and $h_{\widetilde{H}[e]}(z) \subseteq \varepsilon$. Since $(\widetilde{H}, A)$ is a hesitant anti-fuzzy soft commutative ideal over $X$, it follows that

$$
\begin{aligned}
& h_{\widetilde{H}[e]}(x *(y *(y * x))) \\
& \quad \subseteq h_{\widetilde{H}[e]}((x * y) * z) \cup h_{\widetilde{H}[e]}(z) \subseteq \varepsilon .
\end{aligned}
$$

Namely, $x *(y *(y * x)) \in(\widetilde{H}, A)^{\varepsilon}$, and $(\widetilde{H}, A)^{\varepsilon}$ is a commutative ideal of $X$.

Conversely, suppose that $(\widetilde{H}, A)^{\varepsilon}$ is a commutative ideal of $X$ for all $\varepsilon \in P([0,1])$. Put $h_{\widetilde{H}[e]}(x)=\varepsilon$ for any $x \in X$ and $e \in A$. Hence $0 \in(\widetilde{H}, A)^{\varepsilon} \Rightarrow h_{\widetilde{H}[e]}(0) \subseteq \varepsilon=h_{\widetilde{H}[e]}(x)$, for all $e \in A$ and $x \in X$. Now we prove that $h_{\widetilde{H}[e]}(x *(y *(y *$ $x))) \subseteq h_{\widetilde{H}[e]}((x * y) * z) \cup h_{\widetilde{H}[e]}(z)$. In contrast, there exists $x_{0}, y_{0}, z_{0} \in X$ such that

$$
\begin{aligned}
& h_{\widetilde{H}[e]}\left(x_{0} *\left(y_{0} *\left(y_{0} * x_{0}\right)\right)\right) \\
& \quad \supseteq h_{\widetilde{H}[e]}\left(\left(x_{0} * y_{0}\right) * z_{0}\right) \cup h_{\widetilde{H}[e]}\left(z_{0}\right) .
\end{aligned}
$$

Taking

$$
\begin{aligned}
\varepsilon_{0} & =\frac{1}{2}\left\{h_{\widetilde{H}[e]}\left(x_{0} *\left(y_{0} *\left(y_{0} * x_{0}\right)\right)\right)\right. \\
& \left.+\left\{h_{\widetilde{H}[e]}\left(\left(x_{0} * y_{0}\right) * z_{0}\right) \cup h_{\widetilde{H}[e]}\left(z_{0}\right)\right\}\right\},
\end{aligned}
$$

it follows that $h_{\widetilde{H}[e]}\left(\left(x_{0} * y_{0}\right) * z_{0}\right) \subseteq \varepsilon_{0}$ and $h_{\widetilde{H}[e]}\left(z_{0}\right) \subseteq \varepsilon_{0}$, which imply that $\left(\left(x_{0} * y_{0}\right) * z_{0}\right) \in(\widetilde{H}, A)^{\varepsilon_{0}}$ and $z_{0} \in(\widetilde{H}, A)^{\varepsilon_{0}}$. But $(\widetilde{H}, A)^{\varepsilon_{0}}$ is a commutative ideal of $X$, and thus $x_{0} *\left(y_{0} *\right.$ $\left.\left(y_{0} * x_{0}\right)\right) \in(\widetilde{H}, A)^{\varepsilon_{0}}$ or $h_{\widetilde{H}[e]}\left(x_{0} *\left(y_{0} *\left(y_{0} * x_{0}\right)\right)\right) \subseteq \varepsilon_{0}$, and this is a contradiction. Therefore $(\widetilde{H}, A)$ is a hesitant antifuzzy soft commutative ideal of $X$.

\section{Conclusions}

When people make a decision, they are usually hesitant and irresolute for one thing or another which makes it difficult to reach a final agreement. So, we can see that hesitant fuzzy set is a very useful tool to deal with uncertainty. Thus, it is very necessary to develop some concepts about hesitant fuzzy set. Therefore, we introduce the hesitant anti-fuzzy soft set for basic notions in BCK-algebras. In this paper, we introduced the notions of hesitant anti-fuzzy soft subalgebras and hesitant anti-fuzzy soft ideals of BCK-algebras and investigated their relations and properties. However, we defined the notions of hesitant anti-fuzzy soft ideals (implicative, positive implicative, and commutative) in BCKalgebras. Also, we presented the relationship between them and we gave conditions for a hesitant anti-fuzzy soft ideal to be a hesitant anti-fuzzy soft (implicative, positive implicative, and commutative) ideal in BCK-algebras.

\section{Conflicts of Interest}

The authors declare that there are no conflicts of interest regarding the publication of this paper.

\section{Acknowledgments}

This work was supported by Deanship of Scientific Research (DSR), King Abdulaziz University, Jeddah, under Grant no. D-078-363-1437. The authors, therefore, gratefully acknowledge the DSR technical and financial support.

\section{References}

[1] D. Molodtsov, "Soft set theory- first results," Computers \& Mathematics with Applications, vol. 37, no. 4-5, pp. 19-31, 1999.

[2] P. K. Maji, R. Biswas, and A. R. Roy, "Fuzzy soft sets," Journal of Fuzzy Mathematics, vol. 9, no. 3, pp. 589-602, 2001.

[3] L. A. Zadeh, "Fuzzy sets," Information and Control, vol. 8, no. 3, pp. 338-353, 1965.

[4] V. Torra, "Hesitant fuzzy sets," International Journal of Intelligent Systems, vol. 25, no. 6, pp. 529-539, 2010.

[5] K. V. Babitha and S. J. John, "Hesitant fuzzy soft sets," Journal of New Results in Science, vol. 3, pp. 98-107, 2013.

[6] Y. B. Jun, S. S. Ahn, and G. Muhiuddin, "Hesitant fuzzy soft subalgebras and ideals in BCK/BCI -algebras," Scientific World Journal, vol. 2014, Article ID 763929, 2014.

[7] Y. B. Jun and S. S. Ahn, "Hesitant fuzzy sets theory applied to in BCK/BCI- algebras," J. Computational Analysis and Applications, vol. 20, no. 4, pp. 635-646, 2016.

[8] K. Iséki, "An algebraic related with a propositional calclus," Proc. Japan Academy, vol. 42, pp. 26-29, 1996.

[9] J. Meng, Y. B. Jun, and H. S. Kim, "Fuzzy implicative ideals of BCK-algebras," Fuzzy Sets and Systems, vol. 89, pp. 243-248, 1997. 


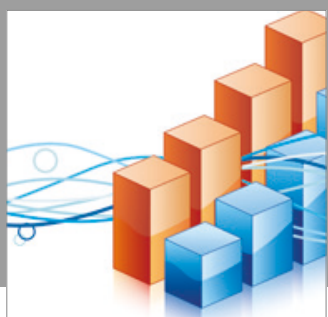

Advances in

Operations Research

vatersals

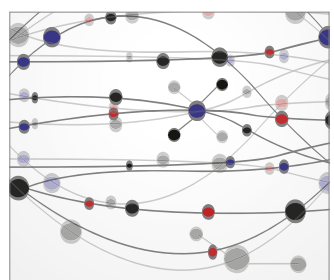

\section{The Scientific} World Journal
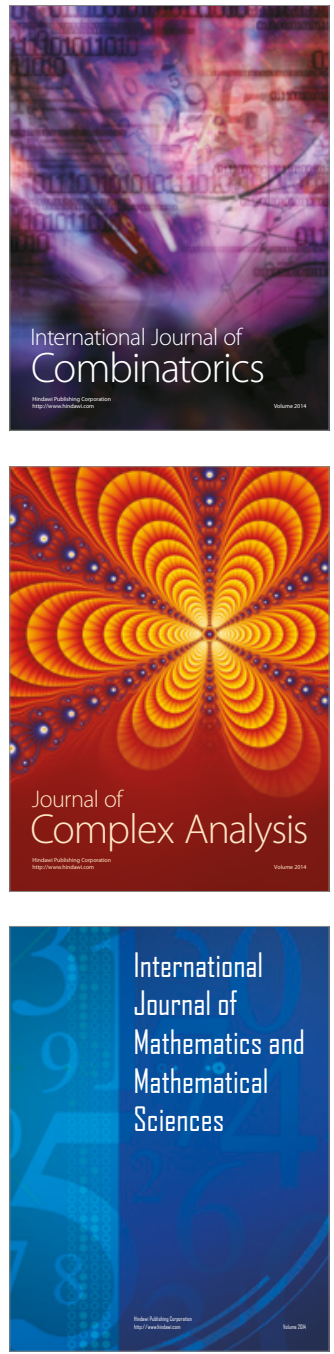
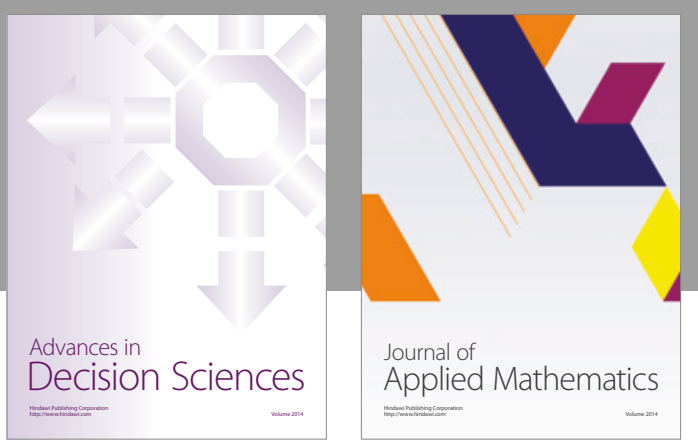

Algebra

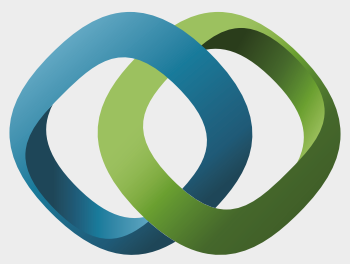

\section{Hindawi}

Submit your manuscripts at

https://www.hindawi.com
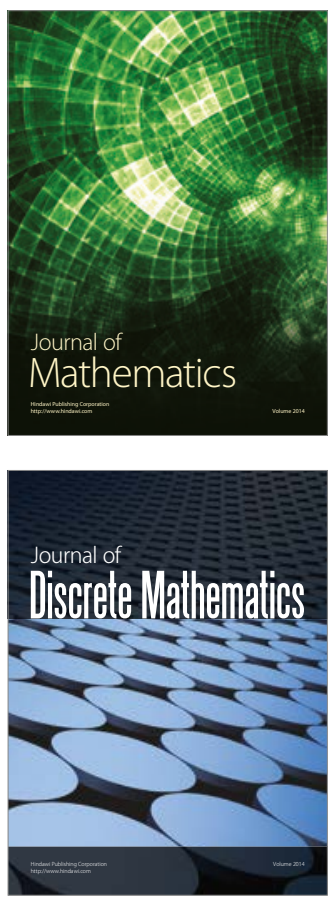

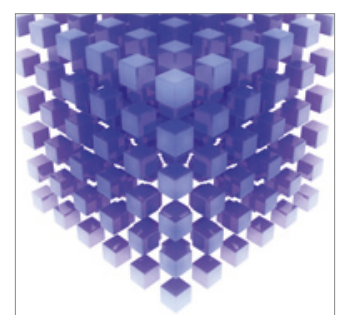

Mathematical Problems in Engineering
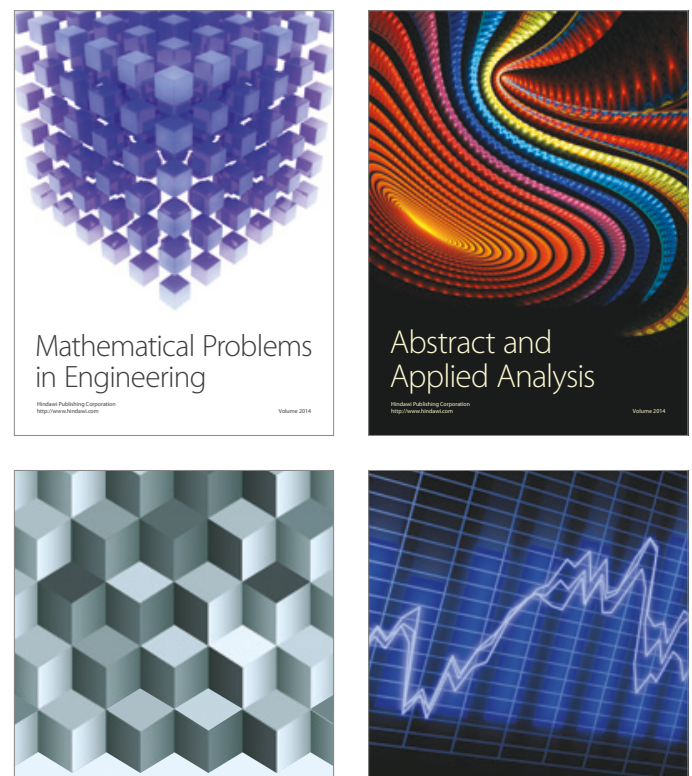

Journal of

Function Spaces

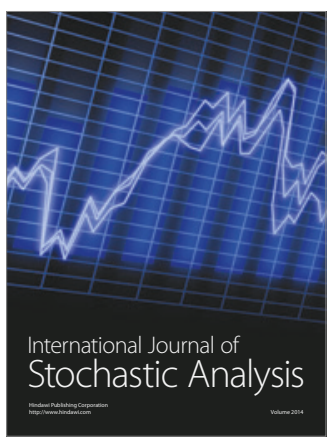

Probability and Statistics
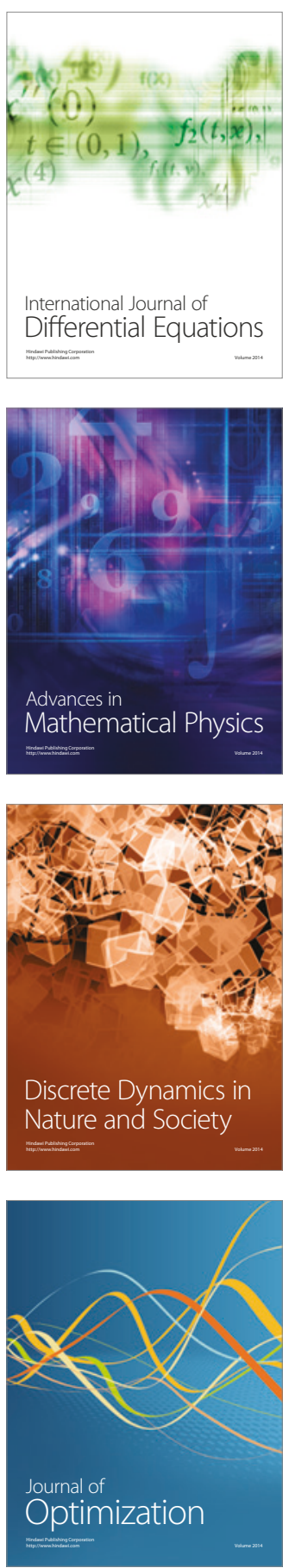